\title{
Envelope analysis of intense relativistic quasilaminar beams in rf photoinjectors: A theory of emittance compensation
}

\author{
Luca Serafini \\ Istituto Nazionale di Fisica Nucleare, Milano, Via Celoria 16, 20133 Milano, Italy \\ James B. Rosenzweig \\ Department of Physics and Astronomy, University of California, Los Angeles, 405 Hilgard Avenue, Los Angeles, California $90095-1547$
}

(Received 11 November 1996)

\begin{abstract}
In this paper we provide an analytical description for the transverse dynamics of relativistic, space-chargedominated beams undergoing strong acceleration, such as those typically produced by rf photoinjectors. These beams are chiefly characterized by a fast transition, due to strong acceleration, from the nonrelativistic to the relativistic regime in which the initially strong collective plasma effects are greatly diminished. However, plasma oscillations in the transverse plane are still effective in significantly perturbing the evolution of the transverse phase space distribution, introducing distortions and longitudinal-transverse correlations that cause an increase in the rms transverse emittance of the beam as a whole. The beam envelope evolution is dominated by such effects and not by the thermal emittance, and so the beam flow can be considered quasilaminar. The model adopted is based on the rms envelope equation, for which we find an exact particular analytical solution taking into account the effects of linear space-charge forces, external focusing due to applied as well as ponderomotive RF forces, acceleration, and adiabatic damping, in the limit that the weak nonlaminarity due to the thermal emittance may be neglected. This solution represents a special mode for beam propagation that assures a secularly diminishing normalized rms emittance and it represents the fundamental operating condition of a space-charge-compensated RF photoinjector. The conditions for obtaining emittance compensation in a long, integrated photoinjector, in which the gun and linac sections are joined, as well as in the case of a short gun followed by a drift and a booster linac, are examined. [S1063-651X(97)10706-1]
\end{abstract}

PACS number(s): 41.75.-i, 41.85.-p, 29.17.+w, 29.25.Bx

\section{INTRODUCTION}

Quasilaminar, space-charge-dominated relativistic electron beams have become a subject of great interest with the advent of short laser pulse-driven radio-frequency (rf) photoinjectors $[1,2]$ that are able to produce electron beams carrying current densities well in excess of $1 \mathrm{kA} / \mathrm{cm}^{2}$, with the transition from the nonrelativistic to the relativistic regime occurring very quickly. The accelerating gradient required to guarantee that the beam will be captured in the rf wave at relevant wavelengths $(5-25 \mathrm{~cm})$ ranges from 10 up to 100 $\mathrm{MeV} / \mathrm{m}$ : the beam is therefore accelerated from rest at the photocathode emissive surface, up to relativistic energy within a fraction of a rf wavelength, which is a distance comparable to one-half of a plasma oscillation period in the transverse plane. The trapping condition is typically expressed as $\alpha>1 / 2$, in terms of the quantity $\alpha$ $=e E_{0} / 2 k m c^{2}$, which represents the dimensionless amplitude of the vector potential associated with the accelerating field, of frequency $\nu_{\mathrm{rf}}\left(k=2 \pi \nu_{\mathrm{rf}} / c\right)$ and amplitude $E_{0}$.

Furthermore, the random, thermal component to the transverse emittance is very small compared to the total rms emittance, which is dominated by the dilution of the projected transverse phase space density due to correlations in the beam distribution function, so that the beam is fairly laminar in both the transverse and longitudinal planes. This implies that in the transverse plane trajectories do not cross each other, while in the longitudinal plane different slices (of length small compared to the total bunch length) do not mix with each other. Since neighboring longitudinal slices additionally do not behave in vastly different ways, precluding the occurrence of large longitudinal density gradients in the beam charge density, this final condition implies that the beam may be broken up, for analysis purposes, into nearly independent longitudinal slices that behave in the same manner as a continuous beam. Evidence for the validity of this model for photoinjector beam dynamics comes from both multiparticle simulations and experiments performed at Brookhaven [3].

This set of conditions, which defines the notion of a quasilaminar beam in this paper, is generally attained in rf photoinjectors, in particular when they are operated in the space-charge-emittance compensation regime [4]. This regime implies that the beam propagates for one transverse plasma oscillation, so that the correlations in the transverse phase space that develop in the first half of the oscillation are undone in the second half by properly focusing the beam. Due to the relativistic diminishing of the space-charge forces as the beam accelerates, one can adiabatically nearly terminate the plasma motion and associated emittance oscillations as the minimum in the emittance occurs, obtaining maximum beam brightness at the exit of the photoinjector.

In this paper we wish to provide a simple framework in which the beam dynamics in such a regime can be analytically described and the space-charge-emittance correction technique can be quantitatively explained. We begin by using a heuristic model of the plasma and emittance oscillations in a quasilaminar beam. This model allows the underlying physical mechanisms involved in the complicated 
phase space dynamics of the rf photoinjector to be elucidated. After this discussion, we then construct the quantitative model for quasilaminar beam propagation. Analytical expressions for the beam envelope from the photocathode surface up to the gun exit in a long, integrated rf photoinjector are provided and the predictions for optimum photoinjector configuration to achieve emittance correction are extracted from the properties of the envelope itself. A particular solution for the beam envelope is found that assures all the bunch slices evolve in transverse space phase with a common phase space angle, which is in fact the desired final state to achieve emittance compensation. This particular solution is termed the invariant envelope, and is in many ways analogous to the equilibrium Brillouin flow of space-chargedominated beams in constant gradient focusing channels. Although this study is directly applied to a description of $\mathrm{rf}$ photoinjectors, the concept of invariant envelope and the method of analysis is of interest and applicable to any relativistic beam that is space-charge-dominated and accelerated in high gradient linear accelerators.

The equation we base our analysis on is Lawson's expression for the evolution of the rms envelope in the paraxial limit [5],

$$
\sigma^{\prime \prime}+\sigma^{\prime}\left(\frac{\gamma^{\prime}}{\beta^{2} \gamma}\right)+K_{r} \sigma-\frac{\kappa_{s}}{\sigma \beta^{3} \gamma^{3}}-\frac{\epsilon_{n}^{2}}{\sigma^{3} \beta^{2} \gamma^{2}}=0
$$

which governs the evolution of the cylindrical symmetric rms transverse beat spot size $\sigma(z)$ under the effects of an external linear focusing channel of strength $K_{r} \equiv$ $-F_{r} / r \beta^{2} \gamma m c^{2}$. Here the prime indicates differentiation with respect to the independent variable $z$, the distance along the beam propagation axis, $\gamma m c^{2}$ is the mean beam energy, and $\beta \equiv \nu_{b} / c=\sqrt{1-\gamma^{-2}}$ is the normalized mean beam velocity. The defocusing space charge term in Eq. (1.1) is proportional to the beam perveance $\kappa_{s}$, and the final term represents the outward pressure due to the normalized rms emittance, which in the case of cylindrical symmetry can be written as

$$
\epsilon_{n}=\beta \gamma \epsilon=\frac{\beta \gamma}{2} \sqrt{\left\langle r^{2}\right\rangle\left\langle r^{\prime 2}\right\rangle-\left\langle r r^{\prime}\right\rangle^{2}}
$$

We use Eq. (1.1) under a host of assumptions, which we now delineate. Equation (1.1) is of course only valid in a paraxial approximation $\left(\sigma^{\prime} \ll 1\right)$ and for a narrow energy spread beam. In our envelope analysis, which is applied only in the region where the beam has attained relativistic velocities (the mean beam velocity $\nu_{b}=\beta c \approx c$ ), the normalized acceleration gradient $\gamma^{\prime}$ is approximated as constant, so that $\gamma\left(z_{2}\right)=\gamma\left(z_{1}\right)+\gamma^{\prime}\left(z_{2}-z_{1}\right)$. In the case of an unbunched beam the perveance takes the form $\kappa_{s}=I / 2 I_{0}$, with $I_{0}$ $=e c / r_{e} \cong 17 \mathrm{kA}$ (for electrons). Since we restrict the discussion to axisymmetric beams, the focusing gradient can incorporate two different types of focusing, that applied externally by a magnetostatic solenoidal focusing field, and the ponderomotive rf focusing [6] produced by the nonsynchronous spatial harmonics of the accelerating rf wave, an effect that is particularly strong in a high gradient standing wave accelera- tor such as an rf photoinjector. As discussed in Refs. [6] and [7], these two focusing sources can be cast into a single expression,

$$
K_{r}=\left[\frac{\eta}{8}+b^{2}\right]\left(\frac{\gamma^{\prime}}{\gamma \sin (\phi)}\right)^{2}
$$

where $b=c B_{z} / E_{0}\left[e E_{0}=2 \gamma^{\prime} m_{e} c^{2} / \sin (\phi), \gamma^{\prime}=\alpha k \sin (\phi)\right]$ for the particular case of a constant solenoidal magnetic field, $\phi$ is the particle phase with respect to the rf field wave, $\phi=\omega t-k z+\phi_{0}$, and $\phi_{0}$ is the rf phase of the bunch centroid at injection. The quantity $\eta$, which is a measure of the higher spatial harmonic amplitudes of the rf wave is defined in Sec. III and is generally quite close to unity in practical rf structures.

We have given the expression for the beam perveance in Eq. (1.1) above for an unbunched beam of constant current I. In our analysis of the quasilaminar beam in Sec. III, we generalize this quantity to include the case of bunched beam, by incorporating a geometrical factor [8] $g(\zeta)$ in the perveance that contains the longitudinal dependence of the transverse space-charge field versus the internal bunch coordinate $\zeta=z-\nu_{b} t$.

Furthermore, Eq. (1.3) ignores possible chromatic aberration effects on the transverse phase space dynamics, due to an energy-phase correlation in the bunch-this analytical study is carried out assuming a monoenergetic bunch. A related source of longitudinal-transverse phase space correlations in this system arises from the phase dependence of the transverse rf forces, which gives rise to an emittance increase [9] at the first iris of the gun (cf. Fig. 3). We assume that this source of emittance, like the chromatic effects, does not give rise to significant changes in the transverse beam dynamics of a given $\zeta$ slice of the bunch. This assumption is quite good, in that these correlations are of a similar form to the those arising from space charge, but smaller in magnitude in nearly all cases of interest. In fact, because of the similarity in spatial dependence of the forces, it has been observed in simulations that the space-charge-emittance compensation process can also partially mitigate this source of emittance [10]. Although the solutions found for Eq. (1.1) can be extended to any kind of charge density distribution in the bunch, the actual predictions of the rf photoinjector designed to achieve emittance compensation will be provided for a density distribution that is Gaussian in all dimensions.

The initial model used for the photoinjector analysis assumes a long multicell rf structure, i.e., an integrated device such as the AFEL (advanced free electron laser) injector at Los Alamos [4], and the proposed PWT (plane wave transformer) injector at University of California at Los Angeles (UCLA) [11]. The analysis is, however, sufficiently broad that many characteristics of photoinjectors with short (one or two cell) rf guns and a postacceleration (booster) linac, where the space-charge compensation takes place in drift space between the rf gun and the booster linac, can be inferred. In fact, the case of a short $(1+1 / 2$ cell $)$ rf gun followed by a drift is discussed in Sec. VI. The exact solution for the beam envelope is not found for this case, but the operating conditions needed to achieve emittance compensation are deduced from the general properties of the envelope equation. 
Radial nonlinearities in the space-charge field are not explicitly taken into account in this model, as they have a weak impact on the rms envelope behavior. The full influence of these effects is beyond the scope of this paper, but is important nonetheless - it is more relevant to a discussion of minimizing the residual emittance after compensation. Some comments on these subjects are made in Sec. II.

In overview, the organization of the paper is as follows. In Sec. II we provide a heuristic model to explain the basics of the emittance oscillation due to a small mismatch of a spacecharge-dominated beam at injection into the focusing channel. Section III is devoted to the detailed analysis of the envelope equation and the model for a multicell photoinjectors; analytical solutions derived in a perturbative approximation around an exact solution are presented. The concept of the invariant envelope is introduced and illustrated in Sec. IV, and its deep relationship with the space-charge-emittance compensation technique is discussed. Predictions relevant to photoinjector design characteristics needed to achieve an invariant envelope operation, i.e., emittance compensation, are presented in Sec. V together with comparisons to numerical simulations of existing rf photoinjector designs. Section VI is devoted to the case of a short rf gun followed by a drift space where the emittance correction takes place. Finally, the implications of the analysis presented in this paper are summarized in Sec. VII.

\section{AN ILLUSTRATIVE MODEL}

The simplified model that we propose for the complicated motion of the beam envelope and emittance evolution in high gradient linear accelerators is motivated primarily by the problem of understanding emittance evolution in rf photoinjector sources. In this model we view the rms emittance as arising from the differing phase space dynamics of each longitudinal slice of the beam, which is assumed to behave as an independent, cold, laminar, space-charge-dominated plasma evolving under the influence of linear external forces. In this case, even though the rms emittance of each longitudinal slice can be neglected in the analysis, the rms emittance of the ensemble can be quite large upon summation of the entire ensemble making up the beam.

In order to understand how this mechanism causes emittance growth, as well as how the emittance growth can be reversed by proper focusing of the beam, we begin by examining a simplified model problem, that of an intense, cold, uniform-density beam nearly matched to an external focusing channel. While this model ignores the effects of acceleration and transverse motion due to the high gradient $\mathrm{rf}$ fields in the accelerator, it serves to illuminate the fundamental dynamics of the emittance oscillations in these devices. When the effects of the high gradient electromagnetic fields are included in the subsequent analysis, analogies to this simple model will be apparent.

We begin by writing the rms envelope equation for a cylindrically symmetric, space-charge-dominated, coasting, relativistic, charged particle beam in a focusing channel of constant strength,

$$
\sigma^{\prime \prime}+K_{r} \sigma=\frac{I}{2 I_{0}(\beta \gamma)^{3} \sigma}+\frac{\epsilon_{n, \text { th }}^{2}}{(\beta \gamma)^{2} \sigma^{3}} .
$$

Given our assumption of space-charge-dominated envelope motion, we may ignore the final term on the right-hand side of Eq. (2.1), which represents the contributions to envelope forcing due to the emittance arising from both random, thermalizing sources as well as the effects of nonlinear macroscopic forces. In this analysis, we will be using Eq. (2.1) to describe the evolution of longitudinal slices of a beam (meaning infinitesimally small lengths $\delta \zeta$ of beam about given values of $\zeta$ ), assuming that the motion of each slice is essentially uncorrelated to that of nearby slices, and in fact depends most strongly only the local (in $\zeta$ ) value of the current. This means that the normalized thermal emittance corresponding to each beam slice is small. We define this emittance formally as

$$
\epsilon_{n, \mathrm{th}}(\zeta) \equiv \frac{\beta \gamma}{2} \sqrt{\left\langle r^{2}\right\rangle_{\zeta}\left\langle r^{\prime 2}\right\rangle_{\zeta}-\left\langle r r^{\prime}\right\rangle_{\zeta}^{2}}
$$

where the subscript $\zeta$ indicates that the average is performed only over the distribution within a given slice.

We next generalize the expression for the space-charge term to include an explicit dependence on the longitudinal position by $I \rightarrow \operatorname{Ig}(\zeta)$, where $I$ is now defined as the maximum current in the beam. The geometrical factor $g(\zeta)$, which is less than unity, is discussed in more detail for a finite beams below; for now let us note that in the limit where the beam is long $\left(\gamma \sigma_{z} \gg \sigma_{r}\right)$ in its rest frame $g(\zeta)$ follows the local dependence of the current very closely.

Upon linearizing Eq. (2.1) about the equilibrium Brillouin flow condition for a slice at a given value of $\zeta$,

$$
\sigma_{\mathrm{eq}}(g(\zeta))=\left(\frac{\operatorname{Ig}(\zeta)}{2 I_{0}(\beta \gamma)^{3} K_{r}}\right)^{1 / 2}
$$

we obtain the equation for small amplitude motion about this point,

$$
\begin{gathered}
\delta \sigma^{\prime \prime}(\zeta)+\left[K_{r}+\frac{\operatorname{Ig}(\zeta)}{2 I_{0}(\beta \gamma)^{3} \sigma_{\mathrm{eq}}^{2} g(\zeta)}\right] \delta \sigma(\zeta)=0 \quad \text { or } \\
\delta \sigma^{\prime \prime}(\zeta)+2 K_{r} \delta \sigma(\zeta)=0
\end{gathered}
$$

which gives oscillation frequencies that are dependent on the external focusing strength, but independent of the beam current. It is this characteristic of the space-charge-dominated, quasilaminar beam dynamics that allows emittance compensation.

This model can be used to illuminate the rf photoinjector case by assuming that the envelopes in the beam ensemble begin (at the "cathode") slightly mismatched to the channel with $\sigma=\sigma_{0}<\sigma_{\text {eq }}$ and $\sigma^{\prime}=0$. All envelope oscillations proceed with the same frequency, given only by the external focusing strength, but with different amplitude and about equilibria that are dependent on the current [if we assume the approximation that $g(\zeta)$ is proportional to the current]. We thus have formally

$$
\sigma(z, \zeta)=\sigma_{\mathrm{eq}}(g(\zeta))+\left[\sigma_{0}-\sigma_{\mathrm{eq}}(g(\zeta))\right] \cos \left(\sqrt{2 K_{r}} z\right)
$$




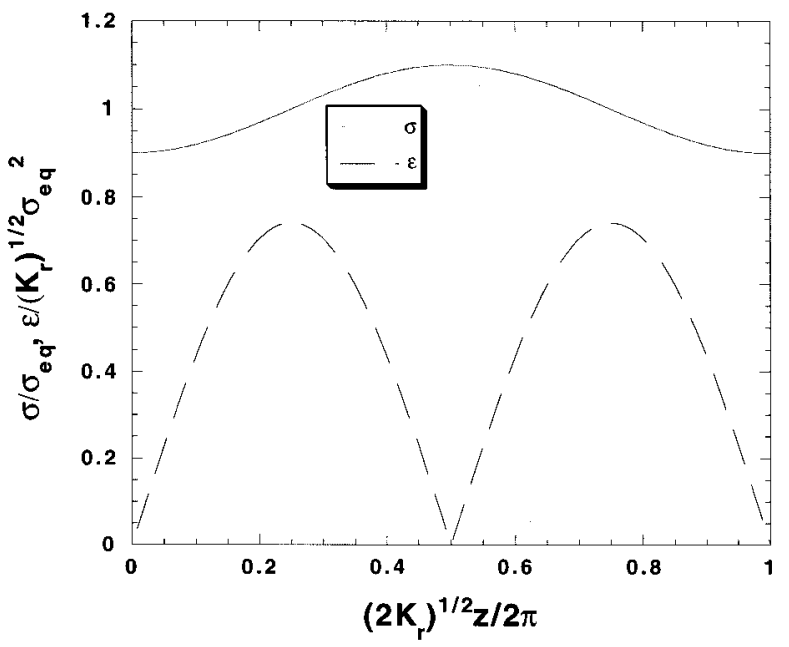

FIG. 1. Emittance and envelope evolution for a slightly mismatched beam ensemble beginning with a minimum beam size and vanishing correlated emittance, in linearized limit.

$$
\sigma^{\prime}(z)=-\sqrt{2 K_{r}}\left[\sigma_{0}-\sigma_{\mathrm{eq}}(g(\zeta))\right] \sin \left(\sqrt{2 K_{r}} z\right) .
$$

Since the frequency of the oscillations is independent of the value of current, but the amplitude is not, the rms emittance of the beam ensemble grows, but returns periodically to minimum values.

This can be seen by noting that under our above assumptions, the rms emittance defined by Eq. (1.2) can be calculated as follows:

$$
\epsilon(z)=\sqrt{\left\langle\sigma^{2}\right\rangle\left\langle\sigma^{\prime 2}\right\rangle-\left\langle\sigma \sigma^{\prime}\right\rangle^{2}},
$$

where the angular bracket indicates an average weighted over the distribution of currents in the entire beam ensemble, i.e., all of the slices. To quantify the effect of the differing trajectories in the ensemble of beam slices, we assume the long beam limit expands the effective distribution function in currents to second order about the maximum current (i.e., near the peak of a symmetric beam current profile that is continuous though its first $\zeta$ derivative), and obtain the emittance evolution

$$
\begin{aligned}
\epsilon(z) & \cong \frac{1}{\sqrt{2}} \sigma_{0}\left(\delta I_{\mathrm{rms}}\right)\left|\frac{\partial}{\partial I}\left(\frac{\sigma^{\prime}}{\sigma}\right)\right|_{I=I_{p}} \\
& \cong \frac{1}{2} \sqrt{K_{r}} \sigma_{0} \sigma_{\mathrm{eq}}\left(I_{p}\right) \frac{\delta I_{\mathrm{rms}}}{I_{p}}\left|\sin \left(\sqrt{2 K_{r}} z\right)\right| .
\end{aligned}
$$

Figure 1 displays the emittance and envelope evolution for a slightly mismatched beam ensemble, beginning, as in the case of the rf photoinjector, with a minimum beam size and vanishing emittance as defined by Eq. (2.7). It can be seen that there are two subsequent emittance null points, one at the maximum in beam size, and another when it returns to its original size. These minima occur where the angles in phase space $\theta=\tan ^{-1}\left(\sigma^{\prime} / \sigma\right)$ are independent of the beam current value. This type of behavior is in fact similar to that observed in rf photoinjectors, as can be seen from the multiparticle simulation shown in Fig. 2, where the beam undergoes one envelope oscillation and two emittance oscillations
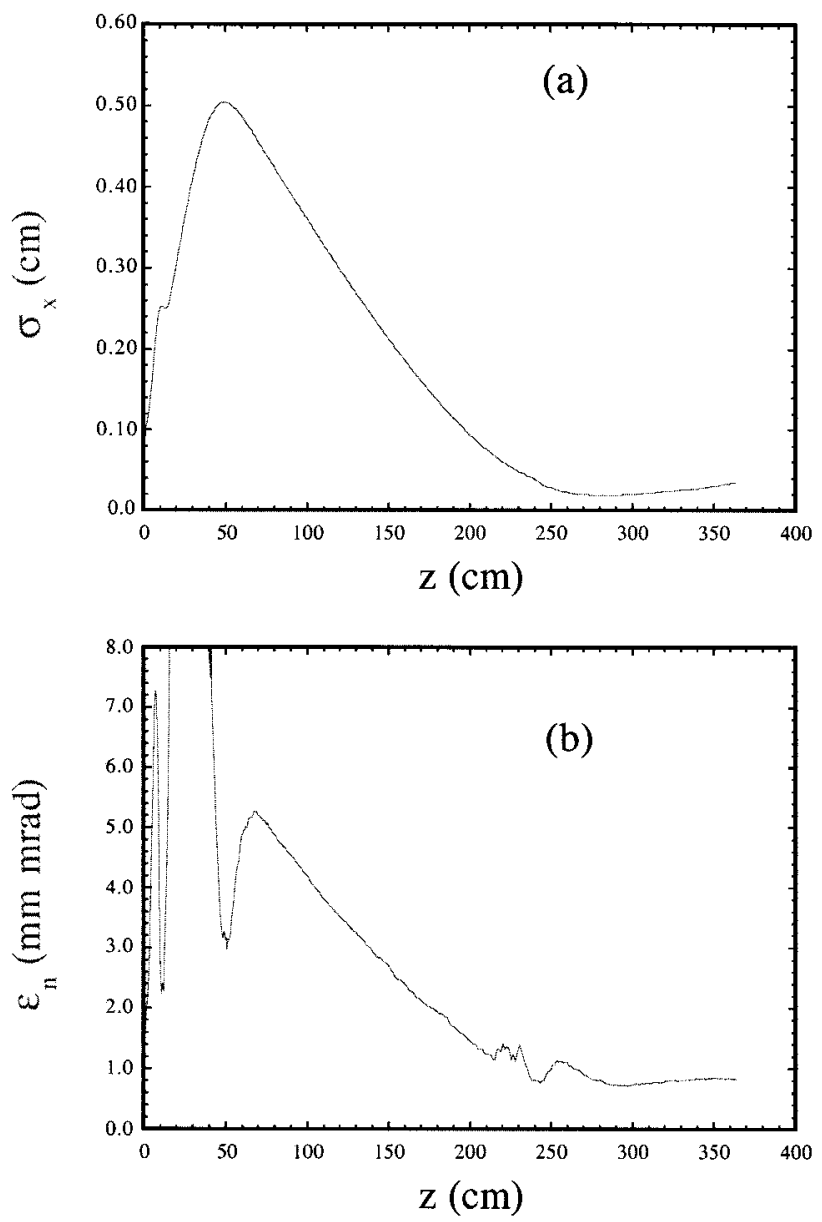

FIG. 2. (a) Envelope, and (b) emittance evolution of $1300-\mathrm{MHz}$ rf photoinjector design, from PARMELA multiparticle simulation.

from the cathode to the injector end in a scaled UCLA Saturnus photoinjector. The emittance minimum occurring at the maximum in the envelope is of secondary interest because it occurs at large beam size and low energy inside of the primary focusing magnet of the rf photoinjector.

The qualitative similarity between the behavior predicted by simple emittance oscillation model and that found in simulation of $\mathrm{rf}$ photoinjectors points the way toward the further analysis of the photoinjector, which differs from the model case in both acceleration and nonuniform application of focusing. One prediction can be gleaned from the simple model even before we begin, which is that one should allow the photoinjector beam to go through only one envelope oscillation, with further oscillations suppressed by diminishing the space-charge forces through acceleration. This must be done with some care, and our analysis leads eventually to a quantitative prescription for obtaining this condition in Sec. V.

This simple model has other aspects that help explain by analogy the behavior of rf photoinjectors operated in the emittance compensation regime. The artifact of the oscillation frequency about the equilibrium being dependent only on the applied restoring force gradient, which is what allows the correlated emittance developing in the beam ensemble to periodically vanish, is not valid to all amplitudes in the beam envelope system. To lowest significant order in the mismatch 


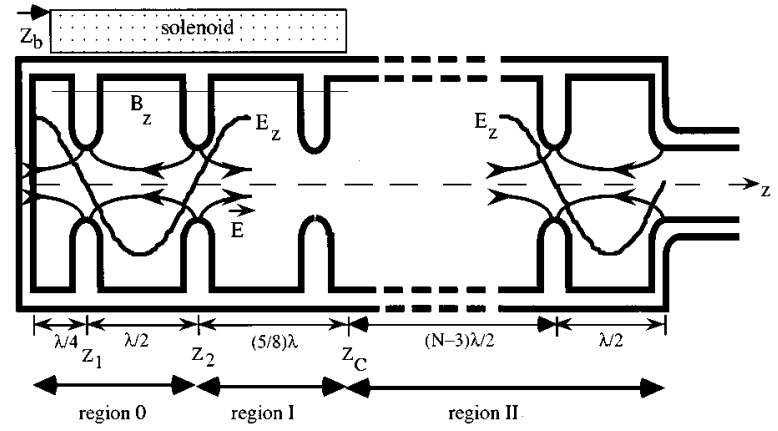

FIG. 3. Schematic cross section in the $(r, z)$ plane of a typical $\mathrm{rf}$ multicell cavity of a photoinjector gun: the rf field distribution on axis is plotted together with the electric field lines of the $\mathrm{TM}_{010-\pi}$ mode in use for electron acceleration. The three different regions $(0$, I, II) used to describe analytically the beam dynamics in the photoinjector are shown.

amplitude $a(I(\zeta))=\left[\sigma_{0}-\sigma_{\mathrm{eq}}(I(\zeta))\right] / \sigma_{\mathrm{eq}}(I(\zeta))$, the oscillation frequency is

$$
\nu(I(\zeta))=\sqrt{2 K_{r}}\left[1+\frac{3}{16} a(I(\zeta))^{2}\right] .
$$

This anharmonicity in the beam ensemble generally precludes the vanishing of the correlated emittance. Thus, there is an additional prediction that can be made on the basis of this observation: excursions in the beam size should be minimized to produce the best emittance compensation.

It should also be noted that, assuming laminarity (there is no "wave breaking" in transverse phase space) within a longitudinal beam slice is maintained, that phase space correlations arising from radial nonlinearities must also behave as do the beam slices-that is, they should also be compensated. Maintenance of laminarity also implies that the beam excursions from equilibrium are limited in amplitude.

\section{BEAM ENVELOPE ANALYSIS FROM CATHODE SURFACE TO INJECTOR EXIT}

In order to perform the envelope analysis of the multicell injector presented below, we must first specify a model for the rf photoinjector. The model we adopt for the accelerating structure is geometry independent, since the accelerating $\mathrm{rf}$ field is written in Floquet form as a sum of its spatial harmonic amplitudes, and the rf photoinjector cavity is assumed to be a multicell structure indefinitely extending along its symmetry axis. There is, however, some specificity in our choice of the model for the static longitudinal magnetic field produced by the external focusing solenoids: it is assumed to have a hard-edge longitudinal profile extending over a few cells of the accelerating structure.

A typical multicell $\mathrm{rf}$ cavity employed in $\mathrm{rf}$ photoinjectors is shown in Fig. 3, displaying the cross section of an axisymmetric iris-loaded structure terminated into a half cell hosting the cathode (located at $z=0$ ) and operated in a $\mathrm{TM}_{010-\pi}$ standing mode with one-half wavelength cells following the cathode cell. The general expression of the rf field components expanded linearly off axis is [6]

$$
\begin{gathered}
E_{z}=E_{0} \sum_{n=1, \text { odd }}^{\infty} a_{n} \cos (n k z) \sin \left(\omega t+\phi_{0}\right), \\
E_{r}=\frac{k r}{2} E_{0} \sum_{n=1, \text { odd }}^{\infty} n a_{n} \sin (n k z) \sin \left(\omega t+\phi_{0}\right), \\
B_{\theta}=c \frac{k r}{2} E_{0} \sum_{n=1, \text { odd }}^{\infty} a_{n} \cos (n k z) \cos \left(\omega t+\phi_{0}\right),
\end{gathered}
$$

where $k=2 \pi / \lambda=\omega / c$, and $a_{n}$ are the spatial harmonic coefficients that depend on the actual cavity geometry that can be computed easily by computer codes or derived by experimental bead measurements. Due to the symmetry of the selected mode, all even $a_{n}$ 's vanish, $a_{1}=1$, and $E_{0}$ becomes the amplitude of the fundamental harmonic (speed-of-light phase velocity) component of the rf wave. All higher harmonic amplitudes are therefore normalized to the value of the fundamental.

The external solenoid is assumed to be folded around the first $2+1 / 2$ cells of the rf cavity, producing a constant magnetic field $B_{z}=B_{0}$ from $z_{b}=\lambda / 8$ (half-way through the cathode cell) up to $z_{c}=11 \lambda / 8$ (a quarter-way through the third full cell). The beam dynamics in the photoinjector are described using a three stage procedure:

(a) The first one and a half cells (from $z=0$ to $z=z_{2}$ ) are treated by using a ballistic approximation, as described in Ref. [7]. In this region, named region 0 , the transverse plasma oscillation begins, driven by the strongly repulsive space-charge forces. The transverse dynamics are dominated by the defocusing effects of space charge and a transient rf kick in the region of the first iris.

(b) In the following cells, i.e., up to the end of the solenoid field at $z=z_{c}$, the envelope equation is solved perturbatively with a constant beam size space-charge approximation. Here, the extra focusing applied by the solenoid field, in conjunction with the ponderomotive $\mathrm{rf}$ focusing, overcomes the transverse space-charge force and turns the beam envelope from divergent to convergent. This is named region I since it is the first region where the envelope equation is applied (and applicable).

(c) In the final region of the accelerator (beyond $z=z_{c}$, named region II), the envelope equation is solved initially as a perturbation about an approximate solution, which provides a general solution to the problem of the beam dynamics up to the end of the photoinjector. In the case of a nearly optimized injector, this approximate solution can be replaced by a special exact solution called the invariant envelope. In this case, the normalized emittance associated with the perturbed plasma oscillations is damped gently for a beam nearly matched to the invariant envelope, while it can be excited to perform additional oscillations if the beam is overfocused by the solenoid, going through successive minima and maxima.

The beam conditions $\sigma_{2}$ and $\sigma_{2}^{\prime}$ at the second iris, i.e., at the end of region 0, are reported in Appendix A $\left(\gamma_{2}=1\right.$ $+3 \pi \alpha / 2$ at $\phi=\pi / 2$ ). With the condition $\alpha>1 / 2$, the trapping threshold requirement that holds for any rf photoinjector [9], the beam at this point is quite relativistic, since typi- 
cally $\gamma_{2} \cong 5-10$. Rewriting the envelope equation assuming $\beta \cong 1$ and beam laminarity (neglecting the thermal emittance), Eq. (1.1) becomes

$$
\sigma^{\prime \prime}+\sigma^{\prime} \frac{\gamma^{\prime}}{\gamma}+\sigma\left(\frac{\eta}{8}+b^{2}\right)\left(\frac{\gamma^{\prime}}{\gamma \sin (\phi)}\right)^{2}=\frac{\kappa_{s}(\zeta)}{\sigma \gamma^{3}},
$$

where the normalized beam energy is given to an excellent approximation by $\gamma=1+\alpha k z \sin (\phi)+\alpha \cos (\phi) \equiv 1+\gamma^{\prime} z$ $+\alpha \cos (\phi)$, and we now leave out the explicit indication of the dependence of $\sigma$ on $\zeta$ and $z$. The ponderomotive rf focusing term displays, through the quantity $\eta$, its dependence on the higher spatial harmonic amplitudes [6],

$$
\eta=\sum_{n=1}^{\infty} a_{n-1}^{2}+a_{n+1}^{2}-2 a_{n-1} a_{n+1} \cos (\phi) \quad\left(a_{0}=0\right) .
$$

In Eq. (3.2) the perveance $\kappa_{s}(\zeta)$ explicitly retains a functional dependence on the longitudinal position $\zeta$ of the particular slice in the bunch, so that $\kappa_{s}(\zeta)=\operatorname{Ig}(\zeta) / 2 I_{0}$. As shown through more detailed calculations in Appendix B, the geometric factor $g(\zeta)$ is given by

$$
g(\zeta)=e^{-\zeta^{2} / 2 \sigma_{z}^{2}}\left(1+\frac{A^{2}}{\gamma^{2}}\left\{\left(1-\frac{\zeta^{2}}{\sigma_{z}^{2}}\right)\left[\frac{1}{2}+\ln \left(\frac{A}{\gamma}\right)\right]-1\right\}\right)
$$

for a Gaussian distribution of aspect ratio $A=\sigma_{r} / \sigma_{z}$, or

$$
g(\zeta)=1-\frac{2 A^{2}}{\gamma^{2}}\left[1+12\left(\frac{\zeta}{L}\right)^{2}+80\left(\frac{\zeta}{L}\right)^{4}\right]
$$

for a uniform distribution of aspect ratio $A=R / L$, where $R$ is the beam radius and $L$ is the beam length. In this section we will assume, for the sake of simplicity, $g(\zeta) \cong I(\zeta) / I_{\text {peak }}$, so that $\kappa_{s}(\zeta)$ does not depend on $\gamma$, which is consistent with the relativistic approximation that the transverse space-chargefield amplitude follows the beam current distribution. As a matter of fact, since the bunch aspect ratio $A$ is typically of the order of 1 , the rest frame aspect ratio is such that $A^{2} / \gamma^{2} \ll 1$ in the domain where Eq. (3.2) is applied ( $\gamma$ $>\gamma_{2}$ ). However, the generalization of Eq. (3.2) to deal with the analysis of bunched beam dynamics will be performed in following sections, together with the analysis of the emittance compensation mechanism.

To solve Eq. (3.2), we apply a Cauchy transformation by changing the independent variable from $z$ to $y$, defined as $y \equiv \ln \left(\gamma / \gamma_{2}\right)$, to obtain

$$
\frac{d^{2} \sigma}{d y^{2}}+\Omega^{2} \sigma=\frac{S(\zeta)}{\sigma} e^{-y},
$$

with $\sigma=\sigma(y)$ and $S(\zeta) \equiv I(\zeta) / 2 I_{0} \gamma_{2} \gamma^{\prime 2}=\kappa_{s}(\zeta) / \gamma_{2} \gamma^{\prime 2}$ defined to be the Cauchy perveance. The transformation clearly reveals, by removing the term $\sigma^{\prime}\left(\gamma^{\prime} / \gamma\right)$ corresponding to adiabatic damping in Eq. (3.2), that the single particle betatron motion in the Cauchy space $(\sigma, y)$ is actually, as long as the space-charge force is neglected, the simple one of a uniform focusing channel with constant normalized focusing gradient $\Omega^{2}$ : this is basically the reason that the matrix derived in Ref. [6] for the transverse motion in rf linacs can be expressed in terms of simple sinusoidal functions. The Cauchy perveance $S$ has been introduced in order to underline the analogy with the usually defined beam perveance, i.e., $I / 2 I_{0} \gamma^{3}$, which turns out to be invariant only for a nonaccelerated beam: before attacking the mathematical treatment of Eq. (3.4) it is worthwhile to anticipate that, since $S$ is invariant in Cauchy space, a sort of equilibrium will be achieved whenever the space-charge term on the right-hand side (RHS) is exponentially damped at the same rate as the combination of focusing and trajectory curvature on the lefthand side (LHS). Since this requires a nonoscillatory behavior for the LHS, which actually represents the betatron motion, this argument leads straight to the requirement of laminarity (i.e., negligible betatron oscillations), which is, consistently, the assumption on which the whole analysis is based.

We obtain solutions to Eq. (3.4) employing two different techniques appropriate to two distinct domains of propagation. As anticipated, we are proceeding from region 0 to I and II by connecting output conditions of each region in input to the following. In region I, defined by $z_{2}<z<z_{c}(0$ $\left.<y<y_{c}\right)$ with $y_{c}=\ln \left\{[1+(5 / 2+1 / 4) \pi \alpha] / \gamma_{2}\right\}$, the beam is exposed to external solenoidal focusing. In this domain we have $\Omega^{2}=\left(\eta / 8+b^{2}\right) / \sin ^{2}(\phi)$, where $b=c B_{0} / E_{0}$. Region II, defined by $z>z_{c}$, is solenoid free and hence $\Omega=\Omega_{0}$ $=(\eta / \sqrt{8}) \sin (\phi)$. In region I the beam size $\sigma$ varies slightly with respect to $\sigma_{2}$, allowing the approximation $\sigma=\sigma_{2}$ in the nonlinear term on the right-hand side of Eq. (3.4). The general solution $\sigma_{\mathrm{I}}$ of the resulting linearized equation is

$$
\begin{aligned}
\sigma_{\mathrm{I}}= & \sigma_{2} \cos (\Omega y)+\dot{\sigma}_{2} \frac{\sin (\Omega y)}{\Omega} \\
& +\frac{S(\zeta)\left[e^{-y}-\cos (\Omega y)+\frac{\sin (\Omega y)}{\Omega}\right]}{\sigma_{2}\left(1+\Omega^{2}\right)},
\end{aligned}
$$

where $\dot{\sigma} \equiv d \sigma / d y$ and $\dot{\sigma}_{2}=\sigma_{2}^{\prime} \gamma_{2} / \gamma^{\prime}$. Setting $\sigma_{c}=\sigma_{\mathrm{I}}\left(y_{c}\right)$ and $\dot{\sigma}_{c}=\dot{\sigma}_{\mathrm{I}}\left(y_{c}\right)$, we can perturbatively solve Eq. (3.4) in region II, assuming that the nonlinear term on the right-hand side may be represented by a particular solution of the form given in Eq. (3.5).

The perturbative solution in the second region $\sigma_{\text {II }}$ then becomes

$$
\begin{aligned}
\sigma_{\mathrm{II}}= & {\left[\sigma_{c}-\frac{S(\zeta) e^{-y_{c}}}{\sigma_{c} \Psi}\right] \cos \left[\Omega_{0}\left(y-y_{c}\right)\right] } \\
& +\frac{S(\zeta) e^{-y+\left(y_{c}-y\right) \dot{\sigma}_{c} / \sigma_{c}}}{\sigma_{c} \Psi} \\
& +\left[\dot{\sigma}_{c}+\frac{S(\zeta) e^{-y_{c}}\left(1+\dot{\sigma}_{c} / \sigma_{c}\right)}{\sigma_{c} \Psi}\right] \sin \left[\Omega_{0}\left(y-y_{c}\right)\right] / \Omega_{0},
\end{aligned}
$$

where $\Psi=\Omega_{0}^{2}+\left(1+\dot{\sigma}_{c} / \sigma_{c}\right)^{2}$. The combination of Eqs. (3.5) and (3.6), together with Eqs. (A4) and (A5), allows the description of the beam envelope from the initial conditions at the photocathode surface up to the photoinjector exit. While this treatment of the behavior of $\sigma_{\mathrm{II}}$ is quite general, it 

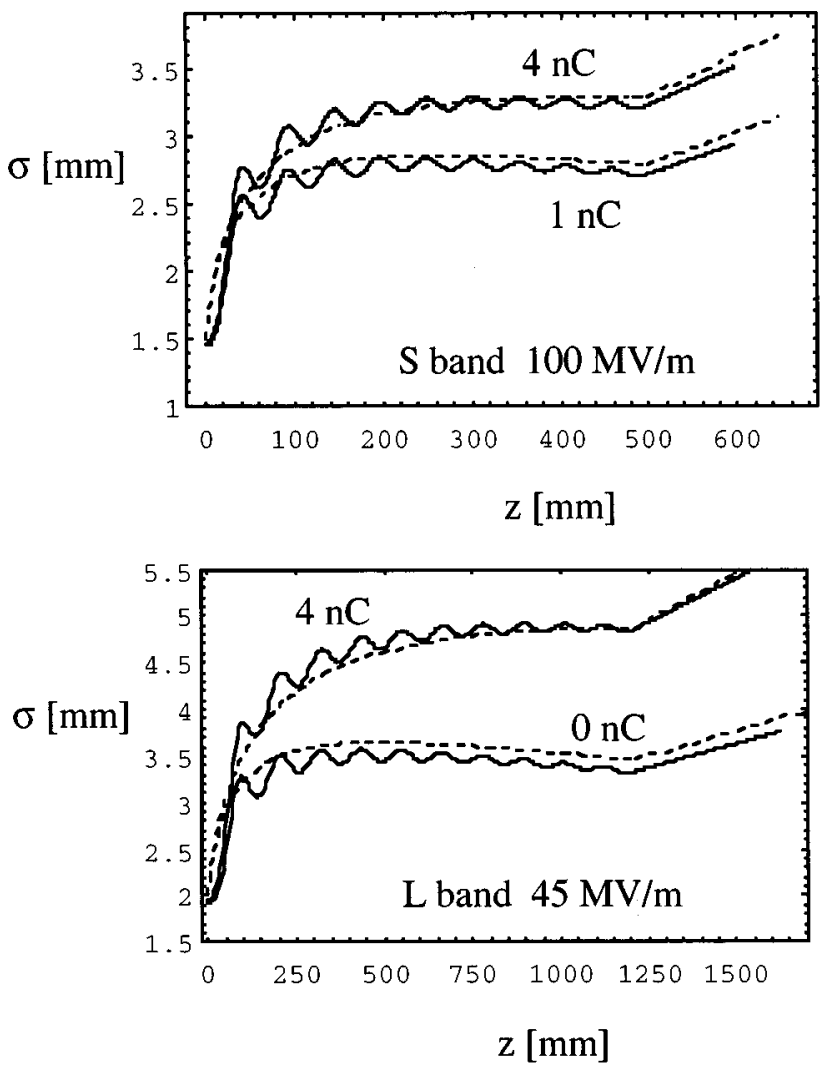

FIG. 4. Beam envelopes through two different $10+1 / 2$ cell $\mathrm{rf}$ guns operated without external solenoid focusing, i.e., $B_{0}=0$ $\left(\nu_{\mathrm{rf}}=2.856 \mathrm{GHz} E_{0}=100 \mathrm{MV} / \mathrm{m}\right.$ upper diagram, $\nu_{\mathrm{rf}}=1.3 \mathrm{GHz} E_{0}$ $=45 \mathrm{MV} / \mathrm{m}$ lower diagram). Dashed lines give the secular orbits analytically predicted, while solid lines are numerical simulation results. Various bunch charges have been used, as indicated.

will ultimately prove less useful than one based on the invariant envelope given in the next section.

The whole system, i.e., the beam and the external RF and magnetic field, can be specified by means of ten operational quantities: the main quantities are the laser pulse characteristics (spot size at the cathode $\sigma_{r}$, pulse length $\sigma_{z} \equiv c \sigma_{t}$ ), the extracted bunch charge $Q_{b}$, the rf field quantities (field amplitude $E_{0}$ and rf frequency $\nu_{\mathrm{rf}}$ ), the magnetic field amplitude of the solenoid $B_{0}$, and the initial and final positions for the solenoid field distribution, namely, $z_{b}$ and $z_{c}$. The somewhat ancillary parameters associated with description of the $\mathrm{rf}$ field are $\eta$ and $\mu$ (defined in Appendix A), which depend on the set of spatial harmonic coefficients $a_{n}$. In the following we will consider the special case of $\eta=\mu=1$, i.e., a pure first harmonic rf field, because it greatly simplifies the analysis without significant loss of generality. In the following we take also $\phi=\pi / 2$, which corresponds to the phase of maximum acceleration.

The beam envelopes resulting from this analysis applied to a multicell photoinjector are shown in Figs. 4 and 5, with several different values of the bunch charge $Q_{b}$ and solenoid field amplitude $B_{0}$ for a typical set of photoinjector parameters. In the upper diagram of Fig. 4 the bunch aspect ratio is $A=1.25$, with $\sigma_{r}=1.5 \mathrm{~mm}$, corresponding to a peak current $I=100 \mathrm{~A}$ at $Q_{b}=1 \mathrm{nC}\left(I=Q_{b} c / \sqrt{2 \pi} \sigma_{z}\right)$ and $400 \mathrm{~A}$ at $Q_{b}$ $=4 \mathrm{nC}\left(z_{2}=79 \mathrm{~mm}, \gamma_{2}=8.7, \alpha=1.64\right)$; in the lower dia-

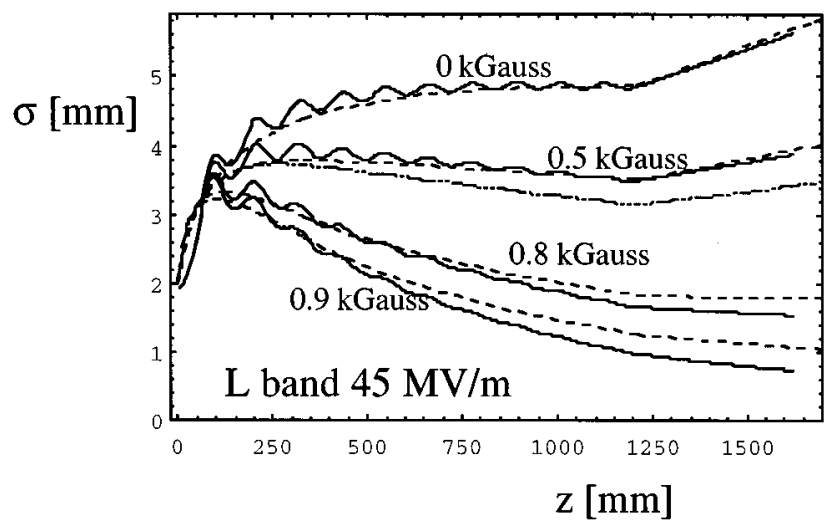

FIG. 5. Beam envelopes through a $10+1 / 2$ cell $L$-band $\mathrm{rf}$ gun $\left(E_{0}=45 \mathrm{MV} / \mathrm{m}, I=200 \mathrm{~A}, Q_{b}=4 \mathrm{nC}\right)$ at different amplitudes $B_{0}$ (in $\mathrm{kG}$ ) of the solenoid magnetic field. Dashed lines give the secular orbits analytically predicted, while solid lines are numerical simulation results.

gram $A=0.83$ with $\sigma_{r}=2.0 \mathrm{~mm}$, giving $I=200 \mathrm{~A}$ at $4 \mathrm{nC}$ $\left(z_{2}=174 \mathrm{~mm}, \quad \gamma_{2}=8.6, \alpha=1.62\right)$ (no solenoid field is present in the data reported in Fig. 4). The simulations were performed with the codes ATRAP [12] for the $S$-band gun (Fig. 4, upper diagram) and ITACA [13] for the $L$-band case (Fig. 4, lower diagram). A similar comparison is shown in Fig. 5, where the extra focusing due to the magnetic field of the solenoid is clearly displayed. It should be noted that by switching off the space charge term in Eqs. (3.5) and (3.6) one obtains the dotted curve plotted in Fig. 5, for the case of $B_{0}=0.5 \mathrm{kG}$, which is clearly mismatched with respect of the simulation curve, indicating the relevance of the nonlinear space-charge term in our analysis.

It is useful to note, as explained in Ref. [6], that a transient angular kick $\Delta \sigma^{\prime}=+\gamma^{\prime} \sigma / 2 \gamma$ (corresponding to $\Delta \dot{\sigma}$ $=+\sigma / 2$ ) must be added to the secular beam envelope at the gun exit in order to transform it back into the actual envelope. What is meant by the distinction secular in describing the envelope is the following: the secular envelope represents the actual envelope averaged over the cell-to-cell oscillations caused by the alternating gradient focusing effect associated with the backward component in the rf standing wave, as discussed in Ref. [6]. The good agreement between the analytically predicted envelopes (dashed lines) and the numerical simulation data (solid lines) gives a significant confirmation of the capability of the present model to predict correctly, within the domain of interest, the beam envelope characteristics.

It is interesting to note that the first two terms on the right-hand side of Eq. (3.5), which scale linearly with the initial conditions $\sigma_{2}$ and $\dot{\sigma}_{2}$, correspond exactly, as far as $b=0$ is set (no superimposed solenoid field), to the linear transport matrix elements derived in Ref. [6] for the evolution of the secular envelope in rf linacs. Therefore, Eq. (3.5) represents the extension of the analysis performed in Ref. [6] to the case of an external magnetic focusing added to the $\mathrm{rf}$ ponderomotive focusing, as well as the contribution from the space-charge field, which is given by the third term on the right-hand side of Eq. (3.5).

\section{THE CONCEPT OF INVARIANT ENVELOPE}

In view of the excellent agreement between the analytical and numerical solutions to the envelope equation for space- 
charge-dominated, strongly accelerating beams in the preceding section, we now extend our analysis to finding a particular beam propagation mode. This mode will be shown to be analogous to the Brillouin flow for space-chargedominated beams in focusing channels discussed in Sec. II.

First of all, we begin by transforming the envelope description in Eq. (3.4) from the Cauchy space $(\sigma, y)$ into a dimensionless Cauchy $(\tau, y)$ space, which displays the fundamental parametric dependence that governs the beam size evolution. By defining the dimensionless quantity $\tau \equiv \sigma / \sqrt{S}$ (we are now, for the sake of compactness, leaving implicit the dependence of $S$ on $\zeta$ ), the envelope equation in the dimensionless Cauchy space $(\tau, y)$ reads

$$
\frac{d^{2} \tau}{d y^{2}}+\Omega^{2} \tau=\frac{e^{-y}}{\tau}
$$

The scaling of the beam size with the square root of the perveance in this analysis naturally agrees with the scaling laws set down in Ref. [17], in that the beam plasma frequency is the same for any envelope of the same $\tau$.

We are interested in the third region $\left(z>z_{c}\right)$, where, taking the case of $\eta=\mu=1$ and $\phi=\pi / 2$, which imply that $\Omega$ $=\Omega_{0}=1 / \sqrt{8}$, the envelope equation reads

$$
\frac{d^{2} \tau}{d y^{2}}+\frac{\tau}{8}=\frac{e^{-y}}{\tau}
$$

which is a universal scaled equation, independent of any external parameter.

Since the quantity $\sqrt{S}$ (which has units of a length) can be related to the transverse plasma frequency $\omega_{p}=\sqrt{4 \pi n_{e} e^{2} / \gamma^{3} m_{e}}=(c / \sigma) \sqrt{I / 2 I_{0} \gamma^{3}}$ by $\omega_{p}=\left(c \gamma^{\prime} / \sigma \gamma\right)$ $\sqrt{S \gamma_{2} / \gamma}$, it is interesting to note that the function $\tau$ can be expressed as $\tau=\gamma^{\prime} e^{-y / 2} / \gamma k_{p}$ $=\left[\left(\gamma^{\prime} / \gamma\right) / k_{p}\right] \sqrt{\gamma_{2} / \gamma}$ with $k_{p}=\omega_{p} / c$. In this form it is clearly shown that $\tau$ scales as the ratio between the local plasma wavelength $\lambda_{p}=2 \pi / k_{p}$, which sets the defocusing length of the beam, and the local incremental energy gain length $L_{g}=\gamma / \gamma^{\prime}$, which sets both the beam adiabatic damping and $\mathrm{rf}$ focusing lengths.

Equation (4.2) has, like Eq. (3.4), a general perturbative solution,

$$
\begin{aligned}
\tau= & {\left[\tau_{c}-\frac{e^{-y_{c}}}{\tau_{c} \Xi}\right] \cos \left[\Omega_{0}\left(y-y_{c}\right)\right]+\frac{e^{-y+\left(y_{c}-y\right) \dot{\tau}_{c} / \tau_{c}}}{\tau_{c} \Xi} } \\
& +\left[\dot{\tau}_{c}+\frac{e^{-y_{c}}\left(1+\dot{\tau}_{c} / \tau_{c}\right)}{\tau_{c} \Xi}\right] \sin \left[\Omega_{0}\left(y-y_{c}\right)\right] / \Omega_{0},
\end{aligned}
$$

with $\Xi=\frac{1}{8}+\left[1+\dot{\tau}_{c} / \tau_{c}\right]^{2}$ and $\Omega_{0}=1 / \sqrt{8}$.

Within this family of solutions there is a notable particular solution,

$$
\hat{\tau}=\sqrt{8 / 3} e^{-y / 2},
$$

corresponding to $\hat{\tau}_{c}=\sqrt{8 / 3} e^{-y_{c} / 2}, \dot{\hat{\tau}}_{c}=-\sqrt{2 / 3} e^{-y_{c} / 2}$, and $\Xi$ $=\frac{3}{8}$. This solution is characterized by having a plasma frequency $\hat{k}_{p}=\sqrt{3 / 2} \gamma^{\prime} / \gamma$, which is proportional to the ponderomotive $\mathrm{rf}$ focusing frequency and (imaginary) adiabatic damping frequency up to a fixed constant. Another way of viewing this is to note that the ratio between the two fundamental scale lengths, $L_{g}$ and $\lambda_{p}$, is in this case exactly constant, i.e., $\hat{\lambda}_{p} / L_{g}=2 \pi \sqrt{2 / 3}$. This is achieved because the scaling of the plasma frequency as $\sqrt{n_{b}} \gamma^{-3 / 2}$ is exactly matched to the energy gain, including the reduction of the beam size $\hat{\sigma}$ with energy, which scales as $\gamma^{-1 / 2}$, namely, $\hat{\sigma}$ $=\left(2 / \gamma^{\prime}\right) \sqrt{I / 3 I_{0} \gamma}$. This constant relationship between $L_{g}$ and $\lambda_{p}$ also explicitly indicates that the invariant envelope is indeed an equilibrium solution in the Cauchy space. Further, it obviously displays the equilibriumlike characteristic that there are no periodic oscillations associated with it, but nearby orbits will oscillate about it; these oscillations will be studied in Sec. V.

The solution $\hat{\tau}$ given by Eq. (4.4) has also the extremely relevant property that it is the only solution displaying a constant phase space angle $\delta(\delta \equiv \dot{\tau} / \tau)$, independent of initial conditions $\tau_{c}$ and $\dot{\tau}_{c}$ in all of the three spaces [Cauchy dimensionless $(\tau, y)$, Cauchy $(\sigma, y)$, configuration space $(\sigma, z)]$. In fact, $\hat{\delta}=\hat{\tau} / \hat{\tau}=\hat{\sigma} / \hat{\sigma}=-1 / 2$, so that in both Cauchy spaces the phase space angle is a universal constant, while in configuration space the phase space angle $\hat{\delta}_{c} \equiv \gamma \hat{\sigma}^{\prime} / \hat{\sigma}=$ $-\gamma^{\prime} / 2$ is a constant (the trace space angle is $\hat{\sigma}^{\prime} / \hat{\sigma}=$ $-\gamma^{\prime} / 2 \gamma$ ). The negative sign of $\hat{\delta}$, implying a convergent beam, is a clear signature of the adiabatic damping due to acceleration: on the other hand, the same quantity is vanishing in the case of Brillouin flow, where [see Eq. (2.3)] $\delta_{\mathrm{Br}}$ $=\gamma \sigma_{\mathrm{eq}}^{\prime} / \sigma_{\mathrm{eq}}=0$.

Further, the most important attribute of $\hat{\delta}$ on the invariant envelope is that it does not depend on the beam current, which is embedded in the perveance scaled variables $\tau_{c}$ and $\dot{\tau}_{c}$. For this reason the solution $\hat{\tau}$ will be called the invariant envelope; its invariance in phase space angle with respect to current is exactly the basic condition to obtain a vanishing linear correlated emittance as the final state of the beam. In fact, it is well known that the emittance growth from linear space-charge effects is due to the angular spread in the phase space distribution of different bunch slices, which receive different kicks from the space-charge field. In analogy to the discussion of the emittance oscillations in the beam mismatched to the solenoid in Sec. II, these different beam slices may be represented by different current amplitudes in Eq. (3.4), with the full beam represented by the ensemble of beam slices. Since Eq. (2.8) predicts emittance oscillations of amplitude scaling like the spread in phase space angles, it is natural to anticipate that this property of the invariant envelope will be crucial to achieve emittance correction, as discussed in detail in Sec. V.

It is interesting to observe that, under the invariant envelope conditions, the space-charge term in Eq. (4.1), $e^{-y} / \hat{\tau}$ $=\sqrt{3 / 8} e^{-y / 2}$, is dominant over the focusing term, which is only one-third of the magnitude of the space-charge term, $\hat{\tau} / 8=(1 / 3) \sqrt{3 / 8} e^{-y / 2}$. Adiabatic damping of the angular divergence due to acceleration provides an additional (damping) term that counteracts the space-charge defocusing in the envelope equation, but it should be noted that the second derivative of the invariant envelope is always positive, thus classifying this trajectory as unstable. This in fact must be the case, since a stable trajectory would imply oscillatory, or nonlaminar, behavior: one of the main consequences of such 
a characteristic of the invariant envelope is the simultaneous damping of the beam spot size $\sigma$ and the beam transverse momentum $p_{\perp} \equiv \gamma \hat{\sigma}^{\prime}=-\gamma^{\prime} \hat{\sigma} / 2$ as $\gamma^{-1 / 2}$.

Since we solve the envelope equation under the assumption of laminarity, the range of validity of such a hypothesis should be investigated. Rewriting Eq. (4.1) by taking into account also the thermal emittance term defined by Eq. (2.2) we find

$$
\frac{d^{2} \tau}{d y^{2}}+\Omega^{2} \tau=\frac{e^{-y}}{\tau}+\left(\frac{2 \epsilon_{n, \text { th }} \gamma^{\prime} \gamma_{2} I_{0}}{I}\right)^{2} \frac{1}{\tau^{3}}
$$

When the beam is on the invariant envelope $\tau=\hat{\tau}$, the second term on the right-hand side of Eq. (4.5) grows as $e^{3 y / 2}$, while the space-charge term decreases as $e^{-y / 2}$. In order to preserve the condition of quasilaminarity, so that the beam can be considered space-charge dominated, the space-charge term must be larger than the emittance term. This condition holds up to a position $y_{l}$ $=\ln \left[\sqrt{(8 / 3) I / 2 I_{0} \epsilon_{n, \text { th }} \gamma^{\prime} \gamma_{2}}\right]$, beyond which the beam enters the region where it becomes emittance dominated. This position corresponds to an energy $\gamma_{l}$ given by

$$
\gamma_{l}=\sqrt{(8 / 3)} \frac{I}{2 I_{0} \epsilon_{n, \mathrm{th}} \gamma^{\prime}} .
$$

Since the thermal emittance $\epsilon_{n, \text { th }}$ is typically of the order of 1 $\mathrm{mm} \mathrm{mrad}$, and taking the relatively high accelerating gradient found in the plane-wave transformer (PWT) linac at UCLA, which is $\gamma^{\prime}=30 \mathrm{~m}^{-1}\left(E_{0}=30.6 \mathrm{MV} / \mathrm{m}\right)$, we have $\gamma_{l}=1.6 I[A]$. This energy is quite a bit larger than that obtained at the UCLA PWT (16 MeV), which, like all existing standing wave photoinjectors, has a peak energy less than 25 $\mathrm{MeV}$, but with peak beam currents in excess of $50 \mathrm{~A}$ considered typical.

Another relevant assumption made above was that of longitudinal laminarity, which means that different slices do not mix with each other. This assumption is not violated in general since, as previously discussed, the longitudinal plasma period is much longer than the typical time scale of emittance compensation (i.e., of one plasma oscillation in the transverse plane). Since the longitudinal plasma frequency, which is suppressed in comparison to the transverse frequency by a geometrical factor $\omega_{p \|} \cong \omega_{p \perp} \sqrt{g\left(\sigma_{z}\right)^{-1}-1}$, where for large beam rest frame aspect ratios $g \approx 1$, the number of plasma oscillations in the longitudinal plane is typically much smaller than 1 . The major result of this longitudinal plasma motion, which, unlike the transverse motion, has little restoring force, is to lengthen the pulse in a laminar fashion; there is relative motion of the beam slices, but they do not overtake each other.

When the beam leaves the accelerating structure one must add a positive (defocusing) kick $\Delta \sigma^{\prime}=+\gamma^{\prime} \sigma / 2 \gamma$, as previously mentioned, to obtain the correct connection between the secular envelope in the gun and the actual envelope outside. Since the corresponding kick in the Cauchy space is $\Delta \dot{\sigma}=+\sigma / 2$, and in Cauchy dimensionless $\Delta \dot{\tau}=+\tau / 2$, it can be clearly seen that a beam propagating through the structure on the invariant envelope, for which $\hat{\tau}=-\hat{\tau} / 2$, will exit the rf structure as a parallel beam, i.e., with $\dot{\tau}=\dot{\sigma}=\sigma^{\prime}=0$ and

$$
\sigma_{f}=\left(2 / \gamma^{\prime}\right) \sqrt{I / 3 I_{0} \gamma_{f}}
$$

where $\gamma_{f}$ is the exit beam energy. This condition is a useful experimental diagnostic of emittance compensation in practice.

The parallel exit condition on the beam envelope points out the analogy between the invariant envelope and the Brillouin flow. In fact the two flows can be matched at the exit of a standing wave linac; equating Eq. (4.7) with Eq. (2.3), one can find that a focusing gradient $K_{r}=\left(\sqrt{3 / 8} \gamma^{\prime} / \gamma_{f}\right)^{2}$ produced by a solenoid of field amplitude $B_{z}=\sqrt{3 / 2} m_{e} c \gamma^{\prime} / e$ can achieve this match, preserving the beam's mean angle in phase space to be vanishing after the linac.

The converse of the exit condition just discussed is the following entrance condition: a beam entering a standing wave linac must have initial beam size given by

$$
\sigma_{i}=\left(2 / \gamma^{\prime}\right) \sqrt{I / 3 I_{0} \gamma_{i}}
$$

with vanishing divergence. In other words, the beam must also enter on a parallel trajectory. The implications of this condition for operation of a split photoinjector, consisting of a short rf gun followed by a drift space and a booster linac, are discussed in Sec. VI.

\section{EMITTANCE COMPENSATION}

External control of the beam spot size and emittance evolution in a long rf photoinjector is accomplished through the variation of the solenoid field strength, which allows one to launch, at $z=z_{c}$, a beam envelope that may be optimized for achieving low emittance performance. It is obvious from the previous section's discussion that this particular envelope solution is of interest from the point of view of emittance control, and so we now concern ourselves with the examination of two issues. The first is how to achieve this "matching", of the beam to the invariant envelope at the end of the solenoid, while the second is the investigation of the subsequent phase space dynamics of a real beam ensemble with a spread in trajectories. Both of these issues are critical in understanding the phenomenon of emittance compensation. We have argued that operation on the invariant envelope is the condition for optimum emittance compensation, in the sense that the beam (the ensemble of all beam slices) fully matched to the invariant envelope displays no further emittance oscillations. It will be shown below that this is only part of the story; beam slices that are not directly on the invariant envelope perform stable oscillations around the invariant envelope, leading to secular damping of the normalized emittance of the full beam ensemble.

At this point, we wish to find proper gun operating conditions, in terms of the six free parameters (spot size at the cathode $\sigma_{r}$, pulse length $\sigma_{z}$, bunch charge $Q_{b}$, rf field amplitude $E_{0}$, frequency $\nu_{\text {rf }}$, and magnetic field amplitude $B_{0}$ ), able to achieve a beam matched at $z=z_{c}$ to the invariant envelope. In order to reduce the number of free parameters we need to specify the matching conditions in the Cauchy dimensionless space, we turn to a set of four free parameters, $\alpha, A, \Lambda$, and $b$, defined by 


$$
\alpha \equiv \frac{e E_{0}}{2 m c^{2} k}, \quad A \equiv \frac{\sigma_{r}}{\sigma_{z}}, \quad \Lambda \equiv \frac{I}{\left(\gamma^{\prime} \sigma_{r}\right)^{2}}, \quad b \equiv \frac{c B_{0}}{E_{0}}
$$

These quantities are physically described as follows: $\alpha$ is the dimensionless amplitude of the rf vector potential, $A$ the bunch aspect ratio, $\Lambda$ the Cauchy current density, and $b$ the magnetic-to-rf focusing ratio. These tuning parameters are linked to the six previous free parameters by $k=2 \pi \nu_{\mathrm{rf}} / c$, $\gamma^{\prime}=e E_{0} / m c^{2}, I=Q c / \sqrt{2 \pi} \sigma_{z}$, while the Cauchy current density $\Lambda$ is given [14] in terms of the current density $J$ by $\Lambda=2 \pi J / \gamma^{\prime 2}$ and is linked to the Cauchy perveance $S$ by $\Lambda=2 I_{0} \gamma_{2} S / \sigma_{r}^{2}$. The merit of the set of four parameters given in Eq. (5.1) consists in the possibility of expressing the beam conditions $\tau_{2}$ and $\dot{\tau}_{2}$ at the exit of the second cell $(z$ $=z_{2}$ ) entirely in terms of these four, as reported in Appendix $A$, instead of the previously used six parameters.

The matching conditions at $z=z_{c}$ can be expressed as

$$
\begin{gathered}
\sqrt{8 / 3} e^{-y_{c} / 2}=\tau_{2} \cos \left(\Omega y_{c}\right)+\dot{\tau}_{2} \sin \left(\Omega y_{c}\right) / \Omega+\frac{\left[e^{\left.-y_{c}-\cos \left(\Omega y_{c}\right)+\sin \left(\Omega y_{c}\right) / \Omega\right]}\right.}{\tau_{2}\left(1+\Omega^{2}\right)} \\
\sqrt{2 / 3} e^{-y_{c} / 2}=\Omega \tau_{2} \sin \left(\Omega y_{c}\right)-\dot{\tau}_{2} \cos \left(\Omega y_{c}\right)+\frac{\left[e^{\left.-y_{c}-\Omega \sin \left(\Omega y_{c}\right)-\cos \left(\Omega y_{c}\right)\right]}\right.}{\tau_{2}\left(1+\Omega^{2}\right)},
\end{gathered}
$$

where $\tau_{2}=\tau_{2}(\alpha, A, \Lambda, b)$ and $\dot{\tau}_{2}=\dot{\tau}_{2}(\alpha, A, \Lambda, b)$ are given by Eq. (A7), while $y_{c}=\ln \{[1+(5 / 2+1 / 4) \pi \alpha] /(1+3 \pi \alpha / 2)\}$ and $\Omega^{2}=\left(1 / 8+b^{2}\right)$. The first two parameters are restricted by practical considerations to a limited range, that is, $0.7 \leqslant \alpha \leqslant 3$ and $1 / 4 \leqslant A \leqslant 2$. Therefore, we solve the two expressions in Eqs. (5.2a) and (5.2b) by expressing their roots as $\Lambda_{s}=\Lambda_{s}(\alpha, A)$ and $b_{s}=b_{s}(\alpha, A)$, which can be well approximated by the expressions

$$
\Lambda_{s}[\mathrm{kA}]=\left[\frac{\sqrt{235-266 \alpha+283 A-188 \alpha A+80 A^{2}-5.3 A^{3}}-15.64-13.2 A}{1.33+0.94 A}\right]^{2}
$$

and

$$
b_{s}=10^{-2}\left[5.7 \alpha-28.5+\frac{77.8+4.8 A}{\alpha^{1 / 4}}-0.15 \Lambda_{s}(\alpha, A)+\sqrt{\Lambda_{s}(\alpha, A)}\left(\frac{5.7}{\alpha^{1 / 4}}-0.84+0.28 A\right)-7.1 A\right] .
$$

These quantities are plotted in Figs. 6 and 7, respectively, as functions of $\alpha$ at different values for $A$, i.e., $A=2,1,1 / 2$, and $1 / 4$. In general, the Cauchy current density $\Lambda_{s}$ increases with $\alpha$ up to a maximum $\alpha_{\max }$ beyond which $\Lambda_{s}$ is no longer defined. The behavior of the values of $b_{s}$ are plotted in Fig. 7 , for the same values of $A$, up to each corresponding $\alpha_{\max }$.

The upper part of the operating diagram in Fig. 6, the region above the dotted line, is in fact forbidden, because in this region the bunch charge is in excess of the maximum, which can be extracted from the cathode surface. This fundamental limitation, as predicted theoretically [15] and observed experimentally [16], sets the maximum achievable current density $J_{\max }$, according to the nonrelativistic ChildLangmuir law, in the form $J_{\max }\left[\mathrm{A} / \mathrm{cm}^{2}\right]=300 \gamma^{\prime} / \sigma_{b}[\mathrm{ps}]$, valid for short bunches $\left(\sigma_{b}[\mathrm{ps}] \ll 1180 / \gamma^{\prime}\right)$. The condition can be cast in terms of $\Lambda_{\max }$ as

$$
\Lambda_{\max }[\mathrm{kA}]=\frac{324}{\alpha \sigma_{\phi}\left[{ }^{\circ} \mathrm{rf}\right]} .
$$

The dotted line plotted in Fig. 6 represents the limitation $\Lambda_{\max }$ for a typical bunch length of $\sigma_{\phi}=2^{\circ}$. Clearly, the optimum operating points should be relatively far from the $\Lambda_{\text {max }}$ line, because of the severe energy spread induced by the longitudinal space-charge field at extraction from the cathode surface (if operating on the line, the photoelectrons in the bunch tail would actually see a vanishing accelerating field at the cathode surface due to the canceling of rf field by the space-charge one). Such a correlated energy spread, i.e., the dependence of energy on the phase or slice position in the bunch, produces chromatic aberrations in the transport through the solenoid field and rf focusing channel, which can prevent the emittance correction process from proceeding correctly. An obvious cure is the use of off-crest acceleration to compensate the space-charge-induced energy spread with an opposite effect from the rf field. In practice, this may imply operation far off crest if the Cauchy current density approaches the limit given by Eq. (5.5).

It is interesting, for the sake of illustration, to plot the current density $J$ and cathode spot size $\sigma_{r}$ corresponding to the line $\Lambda_{s}(\alpha, A=1)$, drawn in the Cauchy operating diagram of Fig. 6, once the rf frequency $\nu_{\mathrm{rf}}=2 \pi \omega$ has been fixed to some representative values, namely, $650 \mathrm{MHz}, 1.3$ $\mathrm{GHz}, 2.856 \mathrm{GHz}$, and $6 \mathrm{GHz}$, as shown in Figs. 8 and 9. As anticipated from the Cauchy operating diagram, each frequency has a definite window in the rf field amplitude in which operation of the injector in the space-chargecompensation regime is possible: the dashed lines set the maximum current density limit corresponding to a bunch length of 5 ps for the upper line and 10 ps for the lower line. The cathode spot size $\sigma_{r}$, plotted in Fig. 9, corresponds to a bunch charge of $1 \mathrm{nC}$; it is given by the relation $\sigma_{r}$ $=\sqrt[3]{(c / \sqrt{2 \pi}) Q_{b} A / \Lambda \gamma^{\prime 2}}$, showing the expected scaling as $Q_{b}^{1 / 3}$ as anticipated in Ref. [17]. 


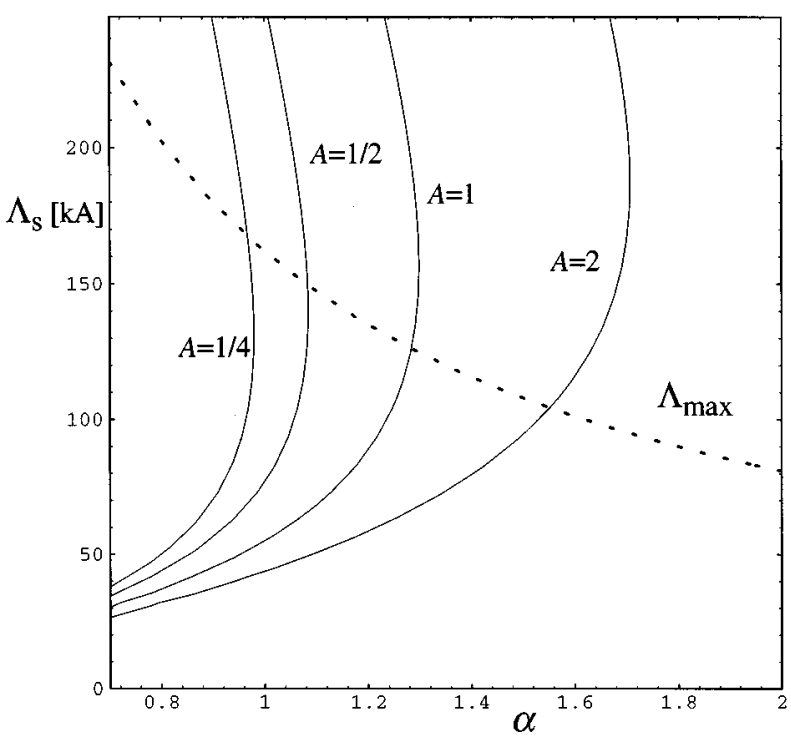

FIG. 6. Operating diagram in the $(\alpha, A)$ plane for an indefinitely long multicell photoinjector.

In order to better illustrate the predictions of the operating diagram in Fig. 6, we choose a point on the diagram, specifically one on the $A=1$ line at $\alpha=1.3$, which corresponds to $\Lambda_{s}=144 \mathrm{kA}$. Choosing the rf frequency to be $1300 \mathrm{MHz}$ ( $L$ band) we obtain $E_{0}=36 \mathrm{MV} / \mathrm{m}$ for the peak cathode field and $\sigma_{r}=0.87 \mathrm{~mm}$ for the cathode spot size and $\sigma_{r}=1.36^{\circ} \mathrm{rf}$ for the bunch length, once we choose the bunch charge $Q_{b}$ to be $1 \mathrm{nC}$. The peak current comes out to be $I=137 \mathrm{~A}$ while $b_{s}=0.85$ implies a magnetic field $B_{0}=1.02 \mathrm{kG}$. The three representative currents, corresponding to three slices, are in this case [cf. Appendix B, Eqs. (B9) and (B10)] $I=137, I^{+}$ $=163$, and $I^{-}=97$. The numerical integration of the envelope equation is shown in Fig. 10(a) (solid lines), where the case for $B_{0}=0$ (no solenoid focusing) is also plotted (dashed lines). The corresponding three slice emittance is shown in Fig. 10(b): as predicted, the normalized emittance is actually corrected only for the case of a beam following the invariant envelope.

We are now in a position to discuss in more detail how the emittance correction process works when the injector is operated under the invariant envelope mode; i.e., it is set at

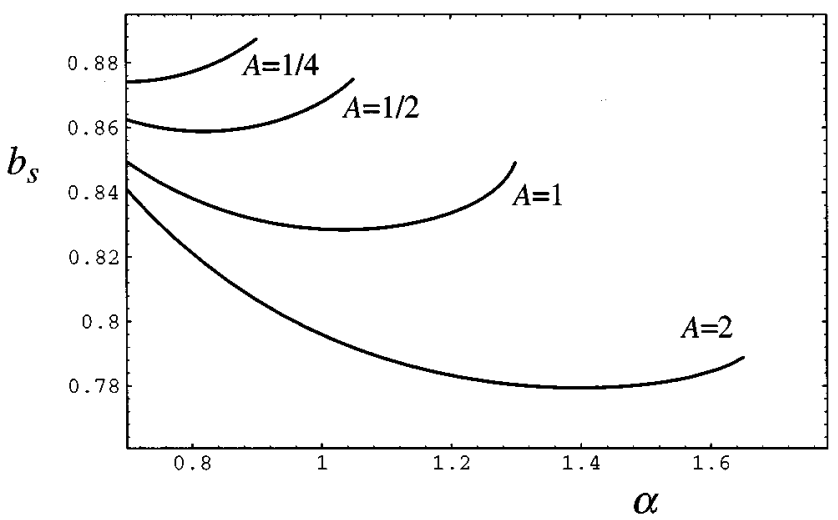

FIG. 7. Parameter $b_{s}$, plotted as a function of $\alpha$, at some values of the bunch aspect ratio $A$, for an indefinitely long multicell photoinjector.

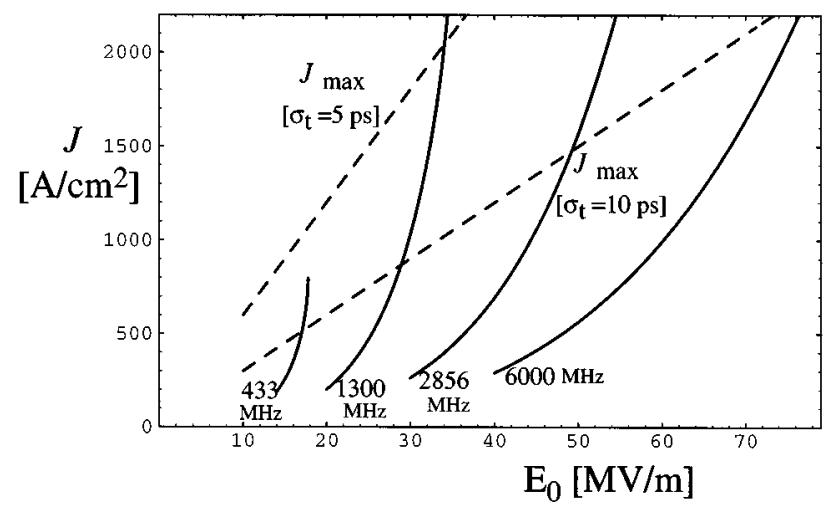

FIG. 8. Current density $J$ plotted (solid lines) vs the cathode peak field $E_{0}[\mathrm{MV} / \mathrm{m}]$, at different $\mathrm{rf}$ frequencies (indicated in $\mathrm{MHz}$ ), for a multicell photoinjector operated in the emittance correction regime. The dashed lines show the limit of maximum $J$ for two different bunch lengths.

the prescribed $\Lambda_{s}$ and $b_{s}$ for a chosen $A$. Let us rewrite Eq. (3.4) in the third region $\left(y>y_{c}\right)$ by explicitly showing the dependence of the Cauchy perveance $S$ and the ponderomotive focusing frequency $\Omega_{0}$ on the slice position $\zeta$ in the bunch:

$$
\left[\frac{d^{2}}{d y_{\zeta}^{2}}+\frac{1}{8 \sin ^{2}(\langle\phi\rangle-k \zeta)}\right] \sigma\left(y_{\zeta}, \zeta\right)=\frac{S(\zeta)}{\sigma\left(y_{\zeta}, \zeta\right)} e^{-y_{\zeta}}
$$

where the Cauchy perveance now becomes $S(\zeta)$ $=\operatorname{Ig}(\zeta) / 2 I_{0} \gamma_{c}(\zeta) \gamma^{\prime}(\zeta)^{2}$, the average accelerating gradient $\gamma^{\prime}(\zeta)=\alpha k \sin (\langle\phi\rangle-k \zeta)$ (with $\langle\phi\rangle$ defined as the bunch average phase), and the initial normalized energy $\gamma_{c}(\zeta)=1$ $+(3 \pi \alpha / 2) \sin (\langle\phi\rangle-k \zeta)+\alpha \cos (\langle\phi\rangle-k \zeta)$. As indicated by the subscript $\zeta$ in the independent variable $y_{\zeta}$, Eq. (5.6) represents actually a family of equations, one for each slice located at a distance $\zeta$ from the bunch central slice, in the variable

$$
\begin{aligned}
y_{\zeta} & \equiv \ln \left[\frac{\gamma(\zeta)}{\gamma_{2}(\zeta)}\right] \\
& =\ln \left[\frac{1+\alpha k z \sin (\langle\phi\rangle-k \zeta)+\alpha \cos (\langle\phi\rangle-k \zeta)}{\gamma_{2}(\zeta)}\right] .
\end{aligned}
$$

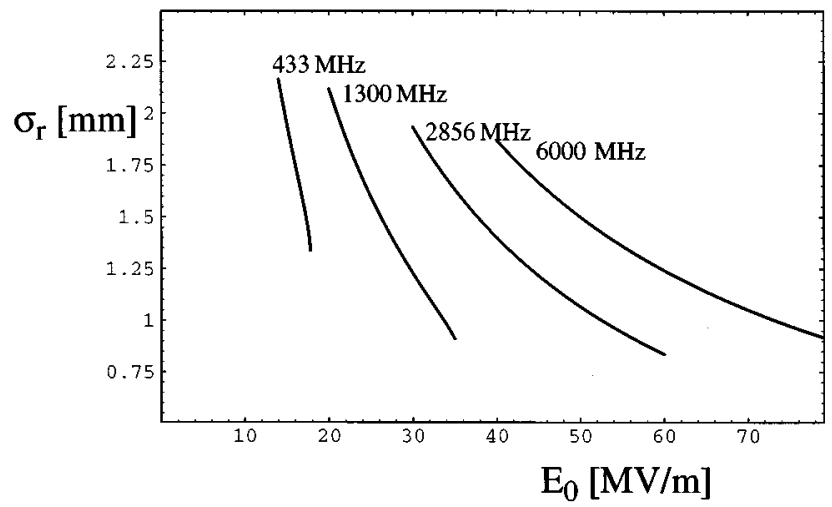

FIG. 9. Cathode spot size $\sigma_{r}$, plotted vs the cathode peak field $E_{0}[\mathrm{MV} / \mathrm{m}]$, at different $\mathrm{rf}$ frequencies, for a multicell photoinjector operated in the emittance correction regime. 

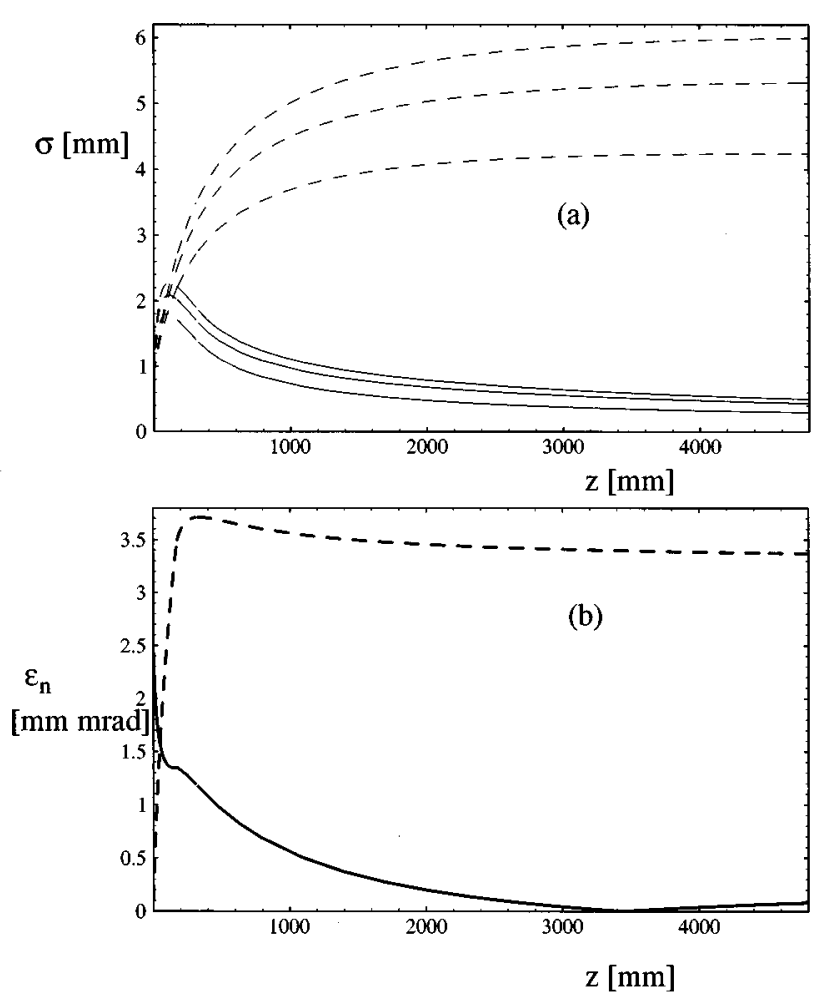

FIG. 10. (a) Envelope and (b) emittance evolution as predicted by the operating diagram in Fig. 6, obtained by numerical integration of the envelope equation, with and without solenoid focusing applied (solid and dashed lines, respectively).

This family of equations can be transformed, in analogy to Eq. (4.2), to read

$$
\left[\frac{d^{2}}{d y_{\zeta}^{2}}+\frac{1}{8 \sin ^{2}(\langle\phi\rangle-k \zeta)}\right] \tau\left(y_{\zeta}, \zeta\right)=\frac{e^{-y_{\zeta}}}{\tau\left(y_{\zeta}, \zeta\right)},
$$

where $\tau\left(y_{\zeta}, \zeta\right) \equiv \sigma\left(y_{\zeta}, \zeta\right) / \sqrt{S(\zeta)}$. The invariant envelope then reads

$$
\hat{\tau}=\frac{2 e^{-y / 2}}{\sqrt{1+1 / 2 \sin ^{2}(\langle\phi\rangle-k \zeta)}},
$$

which in configuration space is simply

$$
\sigma(z, \zeta)=\frac{2}{\gamma^{\prime}(\zeta)}\left(\frac{\operatorname{Ig}(\zeta)}{2 \gamma(\zeta) I_{0}\left[1+1 / 2 \sin ^{2}(\langle\phi\rangle-k \zeta)\right]}\right)^{1 / 2}
$$

As already discussed in Sec. IV, we know that the necessary condition for vanishing correlated emittance growth is that the phase space angles of different slices are equal. In this respect, any effect that induces a correlation, i.e., a $\zeta$ dependence, will produce an emittance increase through the spread of phase space angles of the different slices. The rf effects are basically chromatic, and their phase dependence is typically quite a bit weaker than the dependence of the effective perveance in $\operatorname{Ig}(\zeta)$ in Eq. (5.6), since $\lambda \gg \sigma_{z}$. We thus neglect the chromatic contributions to the dynamics, and concentrate on the charge dependent effects in which the longitudinal correlation of the transverse space-charge field

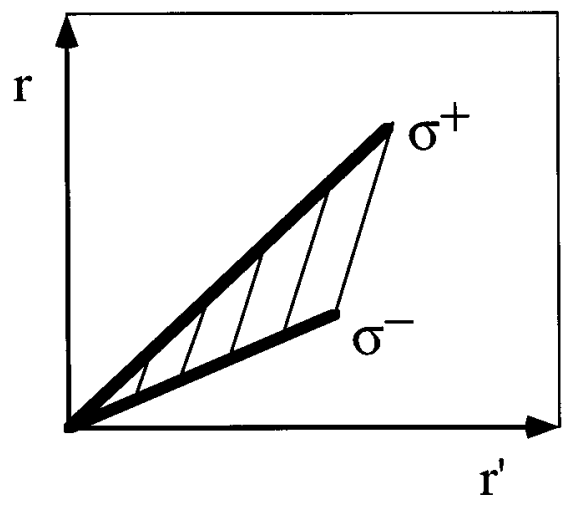

FIG. 11. Description of a bunched beam via two representative slices in the trace space $\left(r, r^{\prime}\right)$.

gives the Cauchy perveance $S$ a slice dependence through the geometrical factor $g(\zeta)$. This condition makes the transformation from Cauchy space $(\sigma, y)$ to the dimensionless Cauchy space $(\tau, y)$ dependent on $\zeta$, an effect that is absent in continuous beams, as previously discussed in Sec. II and analyzed further in Ref. [7]. In this spirit we also set the average phase of the bunch to $\pi / 2$, which corresponds to maximum acceleration. We therefore write Eq. (5.9), under these approximations, as $\sigma(z, \zeta) \cong\left(2 / \gamma^{\prime}\right) \sqrt{\operatorname{Ig}(\zeta) / 3 \gamma I_{0}}$. It obviously is straightforward to generalize the following analysis to include the arbitrary accelerating phase.

Under the assumption of a monoenergetic bunched beam, the Cauchy transformation from $z$ to $y$ is again $\zeta$ independent, and we now write $S=S(\zeta)=\left(I / 2 I_{0} \gamma_{2} \gamma^{\prime 2}\right) g(\zeta)$. We have already shown that the invariant envelope is characterized by a phase space angle independent of the Cauchy perveance $S$, and hence on the current. We now demonstrate that this condition corresponds to a vanishing correlated emittance growth. Since we are dealing with transverse forces that are linear in the radial coordinate, the transverse trace space distribution of the quasilaminar beam $\left(r, r^{\prime}\right)$ is represented by an ensemble of straight segments, one for each slice in the bunch, as depicted schematically in Fig. 11. In this figure only two of these line segments are drawn, one for the central slice (located at $\zeta=0$, having spot size $\sigma_{+}$and divergence $\sigma_{+}^{\prime}$ ), which is subject to the peak space-charge field and another for a slice located at $\zeta=\sigma_{z}$ (with trace space variables $\sigma_{-}$and $\sigma_{-}^{\prime}$ ), where the space-charge field is smaller for typically encountered current distributions, and this reduction is represented by the geometrical factor $g\left(\sigma_{z}\right)<1$. The normalized rms transverse emittance, defined by the relation $\epsilon_{n}(z) \equiv \beta \gamma \epsilon(z) \cong \gamma \epsilon(z)$, with $\epsilon(z)$ given in Eq. (2.7), is explicitly evaluated as

$$
\begin{aligned}
\epsilon_{n}(z) & =\frac{\gamma}{2} \sqrt{\left(\sigma_{+}^{2}+\sigma_{-}^{2}\right)\left(\sigma_{+}^{\prime 2}+\sigma_{-}^{\prime 2}\right)-\left(\sigma_{+}^{\prime} \sigma_{+}+\sigma_{-}^{\prime} \sigma_{-}\right)^{2}} \\
& =\frac{\gamma}{2} \sqrt{\left(\sigma_{+} \sigma_{-}^{\prime}-\sigma_{-} \sigma_{+}^{\prime}\right)^{2}}=\frac{\gamma}{2}\left|\sigma_{+} \sigma_{-}^{\prime}-\sigma_{-} \sigma_{+}^{\prime}\right| .
\end{aligned}
$$

As can be seen from Eq. (5.10), the rms emittance in this two-slice case is identical to the common geometrical defi- 
nition of emittance; it is simply the area of the triangle given by the origin and the two rms phase space points corresponding to the slices.

It should be emphasized at this point that each slice is represented for simplicity by a straight segment in phase space, which is a zero emittance distribution, because we are neglecting the thermal emittance $\epsilon_{n \text {,th }}$, according to our assumption of quasilaminarity. In practice, this emittance, which is added in squares with spatially correlated sources of emittance, can be estimated to be $\epsilon_{n, \text { th }} \approx \sigma_{r} \sqrt{k T / m_{e} c^{2}}$, where the effective (rest frame) temperature $T$ of the beam electrons is determined by the photoemission process, which for metal photocathodes is less than $1 \mathrm{eV}$, and semiconductor cathodes are expected to be one order of magnitude smaller. With these thermal effects, each beam slice's phase space would be a bi-Gaussian distribution whose rms ellipse has an area (the slice emittance) proportional to the thermal emittance $\pi \epsilon_{n, \text { th }}$. The emittance $\epsilon_{n}(z)$ defined by Eq. (5.10) represents a reversible emittance growth that can be corrected by proper beam manipulation, as we are discussing, while the thermal emittance $\epsilon_{n \text {,th }}$ does not arise from reversible transformations and is, in this sense, a true Liouvillian invariant, as discussed in Ref. [15]. It should also be recalled that we are neglecting the emittance due to nonlinear spacecharge fields in this discussion as well.

Assuming for the sake of discussion that the two representative slices follow their own invariant envelopes, we have $\hat{\tau}_{+}=\hat{\tau}_{-}=\hat{\tau}=\sqrt{8 / 3} e^{-y / 2}$, which implies $\sigma_{+}=\left(2 / \gamma^{\prime}\right) \sqrt{\left(I / 3 I_{0} \gamma\right) g(\zeta=0)}$ and $\sigma_{-}$ $=\left(2 / \gamma^{\prime}\right) \sqrt{\left(I / 3 I_{0} \gamma\right) g\left(\zeta=\sigma_{z}\right)}$. For the invariant envelope, we have $\dot{\hat{\tau}} / \hat{\tau}=-1 / 2$, and thus $\sigma_{+}^{\prime}=-\left(\gamma^{\prime} / 2 \gamma\right) \sigma_{+}$and $\sigma_{-}^{\prime}=$ $-\left(\gamma^{\prime} / 2 \gamma\right) \sigma_{-}$. It is readily verified that under these conditions where the normalized emittance defined by Eq. (5.10) vanishes, the invariant envelope is the propagation mode where all the bunch slices are aligned in the transverse phase space.

Clearly, to achieve this ideal beam propagation mode every slice in the bunch must be matched at the invariant envelope, that is, $\sigma_{c}(\zeta) / \sqrt{S(\zeta)}=\hat{\tau}_{c}=\sqrt{8 / 3} e^{-y_{c} / 2}$ $=\sqrt{8 \gamma_{2} / 3 \gamma_{c}} \forall \zeta$. This is an impossible condition to fulfill and, in practice, only a small section of beam can be exactly matched. In this regard, the matching discussed above, dealing with the conditions $\Lambda_{s}$ and $b_{s}$ necessary to operate the photoinjector on the invariant envelope, is clearly a rms matching, because the beam conditions $\tau_{2}$ and $\dot{\tau}_{2}$ [Eqs. (A7) and (A8) ] are given in terms of the rf and space-charge kicks averaged (in the rms sense) over the Gaussian charge distribution. Because of this, only a beam slice equivalent to rms beam is matched; the other beam slices can be considered in general to be mismatched from their invariant envelopes. The dynamics of these mismatched envelopes can be analyzed by perturbation of Eq. (5.6) or its equivalent about the invariant envelope of the matched slice. We thus assume in the following a rms matching and take the equivalent rms beam slice to be $\sigma_{-}=\hat{\sigma}=\hat{\tau} \sqrt{S_{\sigma}}$, with $S_{\sigma} \equiv S_{\text {rms }} \cong S(\zeta$ $\left.=\sigma_{z}\right)$, matched to the invariant envelope $\hat{\tau}$, while $\sigma_{+}=(\hat{\tau}$ $+\delta \tau) \sqrt{S_{0}}$ [with $\left.S_{0} \equiv(\zeta=0)\right]$ is slightly mismatched by a quantity $\delta \tau$ from its invariant envelope.

Substituting $\sigma_{+}$and $\sigma_{-}$in Eq. (5.10), and recalling that $\sigma^{\prime}=\left(\gamma^{\prime} / \gamma\right) \dot{\tau} \sqrt{S}$, we obtain

$$
\begin{aligned}
\epsilon_{n}(z) & =\frac{\gamma}{2}\left|\left(\sigma_{-}+\delta \sigma\right) \sigma_{-}^{\prime}-\sigma_{-}\left(\sigma_{-}^{\prime}+\delta \sigma^{\prime}\right)\right| \\
& =\frac{\gamma^{\prime} S_{\sigma} \hat{\tau}}{2}|\delta \tau+2 \delta \dot{\tau}| .
\end{aligned}
$$

Expressing $\epsilon_{n}(z)$ in terms of physical quantities associated with the invariant envelope, we find that

$$
\begin{aligned}
\epsilon_{n}(z) & \cong \frac{1}{\gamma^{\prime}}\left(\frac{\operatorname{Ig}\left(\sigma_{z}\right)}{3 I_{0} \gamma}\right)^{1 / 2}\left|\delta \sigma \gamma^{\prime}+2 \gamma \delta \sigma^{\prime}\right| \\
& =\frac{\hat{\sigma}}{2}\left|\delta \sigma \gamma^{\prime}+2 \gamma \delta \sigma^{\prime}\right|,
\end{aligned}
$$

where we set $g(0)=1$. We can see that the normalized emittance is proportional to the beam size, which is monotonically decreasing on the invariant envelope; we shall now show that the term inside of the absolute value sign is in fact bounded, and so the emittance also displays a generally monotonically decreasing behavior.

We first study the behavior of deviations from the invariant envelope in Cauchy space, by linearizing Eq. (5.6) around the solution represented by Eq. (5.8) to obtain, for the small-amplitude motion about the invariant envelope

$$
\delta \ddot{\tau}+\Omega^{2} \delta \tau-\frac{e^{-y} \delta \tau}{\hat{\tau}^{2}}=\delta \ddot{\tau}+\left(2 \Omega^{2}+\frac{1}{4}\right) \delta \tau=0,
$$

showing an oscillatory behavior

$$
\begin{gathered}
\delta \tau=\delta \tau_{c} \cos \left[\omega\left(y-y_{c}\right)\right]+\frac{\delta \dot{\tau}_{c}}{\omega} \sin \left[\omega\left(y-y_{c}\right)\right], \\
\delta \dot{\tau}=-\frac{\delta \tau_{c}}{\omega} \sin \left[\omega\left(y-y_{c}\right)\right]+\delta \dot{\tau}_{c} \cos \left[\omega\left(y-y_{c}\right)\right] .
\end{gathered}
$$

for $\delta \tau$ around the invariant envelope with frequency $\omega$ $=\sqrt{2 \Omega^{2}+1 / 4}$, with the constants of integration derived from conditions at $y=y_{c}$. Since $\Omega^{2}=(1 / 8) \sin ^{2}\langle\phi\rangle=1 / 8$, the motion around the invariant envelope is stable, so that any beam injected slightly mismatched to the invariant envelope will follow a trajectory oscillating about it.

This stable motion has, like the small amplitude oscillations discussed in Sec. II, frequency independent of the space-charge strength. This is in fact a general property of the superposition of a linear focusing force with associated frequency $\Omega$ and a repulsive inverse power law force (power $-\alpha$ ), which has a particular equilibriumlike solution, in this case the invariant envelope. The small amplitude oscillations about this particular solution then have frequency $\sqrt{1+\alpha \Omega}$, which depends on the power exponent of the repulsive force, but not its strength, and is always proportional to the linear focusing force strength.

It can be seen in this case that the total potential must exhibit a local parabolic well at the intersection point of the attractive and repulsive force terms. The potential terms that give rise to these forces are shown by the Hamiltonian associated with Eq. (5.7), 


$$
H(y, \zeta)=\frac{p_{\tau}^{2}}{2}+\left[\frac{\tau}{4}\right]^{2}-e^{-y_{\zeta}} \ln \tau, \quad p_{\tau} \equiv \dot{\tau},
$$

in the conjugate variables $\left(\tau, p_{\tau}\right)$. The Hamiltonian $H$ is not a constant of the motion, as indicated by the explicit dependence on the independent variable $y$. The perturbed Hamiltonian, however, is a constant for small amplitude motion about the invariant envelope. The resultant simple-harmonic small amplitude motion can be seen to be manifestly Liouvillian, and the $\left(\tau, p_{\tau}\right)$ phase space area (an emittance, which we discuss further below) is a constant of the motion as well. This fact guarantees that the normalized emittance must damp as $\gamma^{-1 / 2}$, as illustrated by the two-point emittance given by the second form of Eq. (5.11).

To further illustrate these points, it is perhaps more instructive to view the oscillations around the invariant envelope in physical variables at this point. The physical space analogue to Eq. (5.13), which describes oscillations about the invariant envelope can be written as

$$
\delta \sigma^{\prime \prime}+\left(\frac{\gamma^{\prime}}{\gamma}\right) \delta \sigma^{\prime}+\frac{1}{2}\left(\frac{\gamma^{\prime}}{\gamma}\right)^{2} \delta \sigma=0 .
$$

This equation has the general solution

$$
\begin{gathered}
\delta \sigma=\delta \sigma_{c} \cos (\psi)+\sqrt{2}\left(\frac{\gamma_{c}}{\gamma^{\prime}}\right) \delta \sigma_{c}^{\prime} \sin (\psi), \quad \text { with } \\
\delta \sigma^{\prime}=-\frac{1}{\sqrt{2}}\left(\frac{\gamma^{\prime}}{\gamma}\right) \delta \sigma_{c} \sin (\psi)+\delta \sigma_{c}^{\prime}\left(\frac{\gamma_{c}}{\gamma}\right) \cos (\psi),
\end{gathered}
$$

$$
\begin{aligned}
\epsilon_{n}(z) & =\gamma\left\{\left\langle\delta \sigma^{2}\right\rangle\left\langle\delta \sigma^{\prime 2}\right\rangle-\left\langle\delta \sigma \delta \sigma^{\prime}\right\rangle^{2}+\hat{\sigma}^{2}\left[\left\langle\delta \sigma^{\prime 2}\right\rangle+\frac{\gamma^{\prime}}{\gamma}\left\langle\delta \sigma \delta \sigma^{\prime}\right\rangle+\left(\frac{\gamma^{\prime}}{2 \gamma}\right)^{2}\left\langle\delta \sigma^{2}\right\rangle\right]\right\}^{1 / 2} \\
& =\left\{\epsilon_{\mathrm{off}}^{2}+\hat{\sigma}^{2}\left[\left\langle\left(\gamma \delta \sigma^{\prime}\right)^{2}\right\rangle+\gamma^{\prime}\left\langle\delta \sigma\left(\gamma \delta \sigma^{\prime}\right)\right\rangle+\left(\frac{\gamma^{\prime}}{2}\right)^{2}\left\langle\delta \sigma^{2}\right\rangle\right]\right\},{ }^{1 / 2}
\end{aligned}
$$

where we have defined the normalized offset emittance of the distribution,

$$
\epsilon_{\mathrm{off}} \cong \gamma \sqrt{\left\langle\delta \sigma^{2}\right\rangle\left\langle\delta \sigma^{\prime 2}\right\rangle-\left\langle\delta \sigma \delta \sigma^{\prime}\right\rangle^{2}},
$$

and the indicated averages are over the $n$-slice distribution. The normalized offset emittance is a constant of the motion; it can be evaluated, for example, at the beginning of invariant envelope propagation as $\epsilon_{\text {off }}$ $\cong \gamma \sqrt{\left\langle\delta \sigma_{c}^{2}\right\rangle\left\langle\delta \sigma_{c}^{\prime 2}\right\rangle-\left\langle\delta \sigma_{c} \delta \sigma_{c}^{\prime}\right\rangle^{2}}$. It is also clear from Eqs. (5.17) that the term inside the square brackets in Eq. (5.19) is bounded and oscillatory. Therefore we can write the general form of emittance evolution as

$$
\epsilon_{n}(z)=\sqrt{\epsilon_{\mathrm{off}}^{2}+\hat{\sigma}^{2}\left(a+b \cos ^{2}\left[\psi+\theta_{c}\right]\right)},
$$

where $a, b$, and $\theta_{c}$ are constants describing the orientation of the offset distribution, and $\psi$ is as defined below Eq. (5.17). where $\psi \equiv(1 / \sqrt{2}) \ln \left(\gamma / \gamma_{c}\right), \delta \sigma_{c}=\sigma_{c}-\left(2 / \gamma^{\prime}\right) \sqrt{I / 3 I_{0} \gamma_{c}}$, and $\delta \sigma_{c}^{\prime}=\sigma_{c}^{\prime}+\sqrt{I / 3 I_{0} \gamma_{c}^{3 / 2}}$ for the mismatched (core) envelope. It can be seen that the determinant of the matrix of the ( $\left.\delta \sigma, \delta \sigma^{\prime}\right)$ transformation is simply $\gamma_{c} / \gamma$, which is expected from adiabatic damping of the transverse oscillations. Thus we see that the normalized offset emittance associated with the phase space of the perturbed oscillations centered on the invariant envelope is conserved. This is to be expected from the Liouvillian nature of the perturbed envelope system.

Before discussing a general distribution, we first examine the behavior of the two-slice case introduced in Eqs. (5.12). In this case the emittance is given by

$$
\begin{aligned}
\epsilon_{n}(z) \cong & \frac{1}{\gamma^{\prime}}\left(\frac{\operatorname{Ig}\left(\sigma_{z}\right)}{3 I_{0} \gamma}\right)^{1 / 2} \mid\left(\delta \sigma_{c} \gamma^{\prime}+2 \delta \sigma_{c}^{\prime} \gamma_{c}\right) \cos (\psi) \\
& +\left(\delta \sigma_{c}^{\prime} \gamma_{c}-\delta \sigma_{c} \gamma^{\prime}\right) \sqrt{2} \sin (\psi) \mid
\end{aligned}
$$

Equation (5.18) shows the expected $\gamma^{-1 / 2}$ damping of the normalized emittance, with anharmonic oscillations of periodicity 2 times shorter than the period of the perturbations about the invariant envelope.

For the case of a general $n$-slice distribution, with a symmetric spread in mismatch amplitude about the invariant envelope, it can be shown by extending the above arguments that the normalized emittance that is projected by this distribution of phase space orbits offset from the origin in phase space has the form 
A schematic picture of the phase space of a beam that is matched in the rms sense to the invariant envelope is shown in Fig. 12. For the sake of illustration, the familiar form of an ellipse is used to indicate the offset phase space distribution boundary. This ellipse has an invariant area $\pi \epsilon_{\text {off }}$, and rotates with the same frequency as the envelope oscillations, $\omega=4 \omega_{p} / \sqrt{3}$. Figure 12 illuminates, by a phase space diagram, the physical mechanism of emittance compensation; reassuringly, there is a Liouvillian space of orbits about the invariant envelope with conserved phase space area-the phase-space-centered rms emittance of the beam damps as the offset from the origin $\left(\hat{\sigma}, \hat{\sigma}^{\prime}\right)$ of the distribution approaches the origin in phase space. The normalized offset emittance can be therefore thought of as a strict lower bound on the phase-space-centered normalized emittance of the distribution. One cannot actually extrapolate the damping of the emittance to this level, however, as this would violate the assumption of quasilaminarity, which requires that the offset be larger than the spread of beam sizes in the distribution. This argument implies the normalized emittance must be several times larger than $\epsilon_{\text {off }}$, when the emittance compensation process is halted by nonlaminar (crossover) beam trajectories.

As a final note on the dynamics of emittance compensation in a long accelerating structure, we point out that as the beam exits the focusing solenoid, it has just passed its maximum in beam size and local minimum in emittance. The emittance would, in the absence of acceleration, tend to rise again, but this rise is held in check by the $\gamma^{-1 / 2}$ damping effects predicted by Eqs. (5.18)-(5.21). The emittance tends not to damp much for one-quarter of a perturbed beam (plasma) oscillation after this point, because the orientation of the offset ellipse major axis moves towards normal to the nominal phase space angle of the invariant envelope. Only after the ellipse major axis begins rotating back towards alignment with the average phase space angle does the emittance damping become more apparent, as has been deduced from multiparticle simulations. In fact, this rotation tends to occur after the beam exits the accelerating structure, because the phase advance of the plasma oscillation is slowed by the acceleration process. This can be quantified as follows: the phase advance between where the beam is focused onto the invariant envelope (the exit of the solenoid) and where the emittance minimum occurs must be between one-quarter and one-half of a wavelength, or $\pi / 2<(1 / \sqrt{2}) \ln \left(\gamma / \gamma_{c}\right)<\pi$ [recalling that the phase advance is defined as $\psi$ $\left.\equiv(1 / \sqrt{2}) \ln \left(\gamma / \gamma_{c}\right)\right]$. This gives a multiplication of the energy beyond the solenoid of between 9.2 and 85 . Even the smaller of these numbers implies a structure over 25 cells long, which is longer than any integrated photoinjector yet built by a factor of 2. Thus the final compensation must occur within the drift space after the exit of the accelerating structure, where the beam is small (and nearly parallel, as is discussed in Sec. VII), and its plasma frequency nearly constant, instead of diminishing as $\omega_{p} \propto \gamma^{-1} \propto z^{-1}$ in the accelerating case.

These effects are illustrated well by a PARMELA simulation of a new $11+1 / 2$ cell, $2856-\mathrm{MHz}$ rf photoinjector currently under design at UCLA. In this case, a solenoid field, as calculated by the magnetostatic simulation code POISSON, is localized in the initial 2.5 cells of the structure to closely

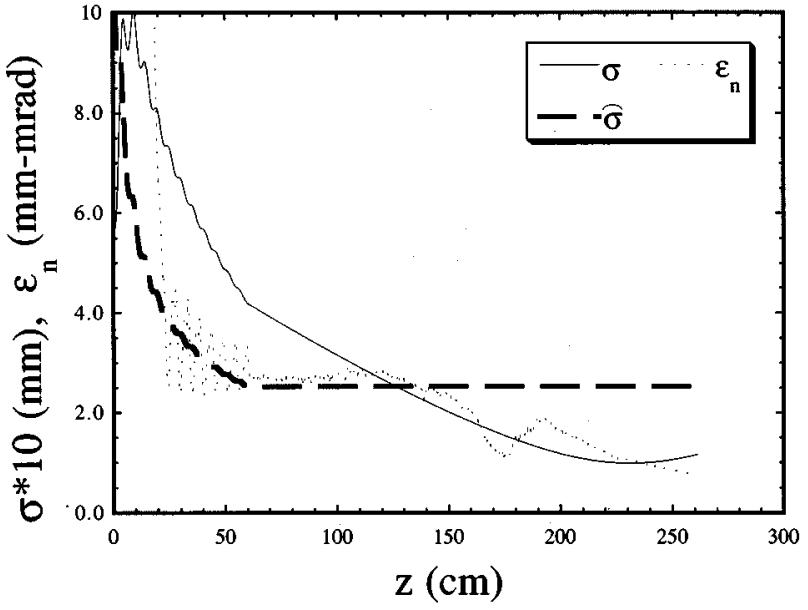

FIG. 13. PARMELA simulation of $11+1 / 2$ cell, 2856-MHz rf photoinjector currently under design at UCLA. The solenoid field is localized in the initial 2.5 cells of the structure, $Q=1 \mathrm{nC}$ and $\sigma_{z}$ $=0.78 \mathrm{~mm}(I=111 \mathrm{~A})$, and $E_{0}=60 \mathrm{MeV} / \mathrm{m}$. Resulting evolution of rms emittance, and beam envelope, as well as the invariant envelope are plotted. Compensation occurs far after the photoinjector exit.

approximate the analytical model we have used. The beam charge is $1 \mathrm{nC}$ and the rms bunch length is $0.78 \mathrm{~mm}$, with an associated current of $111 \mathrm{~A}$, and the peak acceleration gradient on axis in this structure is $60 \mathrm{MeV} / \mathrm{m}(\alpha=0.98)$. The evolution of the rms emittance, the rms beam envelope, and the invariant envelope obtained from this simulation are shown in Fig. 13. It can be seen that the beam envelope is close to the invariant envelope in the photoinjector, but slightly larger and more convergent. After the photoinjector exit the beam envelope converges to below the invariant envelope, and compensation of the emittance takes an additional $2 \mathrm{~m}$ drift. The convergence of the beam to a denser focus in the drift also serves to increase the plasma frequency and decrease the length to compensation.

This example illustrates that one must be careful in interpreting the $\gamma^{-1 / 2}$ dependence of the emittance evolution found in the above analysis. It may be more illustrative to cast the assertion in a different light, by stating that the emittance scales as $\sigma$, and that the invariant envelope propagation is the generalized equilibrium mode that must be followed to achieve this scaling with bunch size.

\section{PHOTOINJECTORS WITH COMPACT GUNS AND BOOSTER LINACS}

While the long rf photoinjector analyzed thus far is encountered in practice, with the noted examples of the LANL (Los Alamos National Laboratory) photoinjectors APEX (advanced photoinjector experiment) and AFEL, it is much more common experimentally to employ a compact rf gun ( $N+1 / 2$ cells, $N \leqslant 3$ ) followed by a drift space and a booster linac. In this configuration, the beam is focused near the end of the gun by a strong solenoidal field. The beam then drifts after focusing, undergoing a diminishing phase of an emittance oscillation as the beam becomes smaller, eventually minimizing as a space-charge-dominated beam waist is reached. The booster linac entrance is placed at this point to 

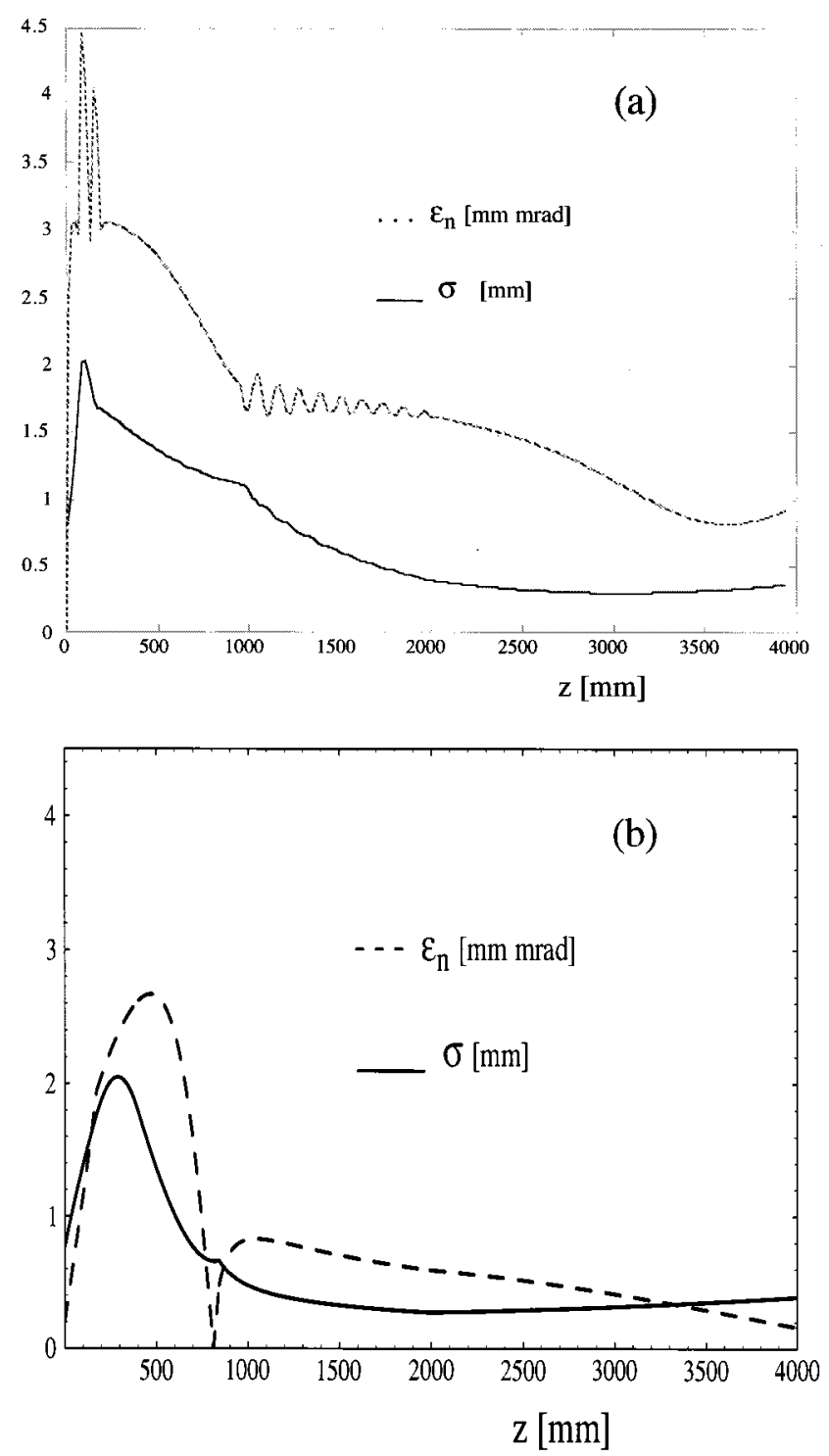

FIG. 14. (a) Envelope and emittance evolution of the beam in the TTF-FEL photoinjector as obtained from particle-in-cell simulation. The analytical prediction of the correct invariant envelope in this case is shown for comparison in (b).

begin acceleration, extending further the process of emittance compensation. This waist should be chosen to both give a small emittance at the waist point and to match onto the invariant envelope associated with the beam current and energy, as well as the linac accelerating gradient. An illustrative example of such a system is shown in Fig. 14(a), which displays the rms envelope and emittance evolution of the beam in the TTF-FEL (Tesla Test Facility-free electron laser) photoinjector as obtained from a particle-in-cell simulation performed with ITACA [13]. The analytical prediction of the correct invariant envelope in this case is shown for comparison in Fig. 14(b). It is very close to this optimization found by performing many such simulations, thus validating the approach to photoinjector design we have deduced from this analysis.

The number $N$ of full cells in the gun is a variable in this analysis, but the validity of the approach followed here is confined to a few cells. The most commonly encountered case in practice, of course, is one full cell. We will also consider, as a particular case, the possibility to slightly vary the length of the first half cell, as it is known from experience that a slightly longer (typically 0.625 instead of 0.5 ) first cell gives better performances in terms of emittance correction. This generalization, while a departure from the model employed in the previous sections, is necessary for an accurate comparison of the theory to actual rf photoinjector configurations.

The basic strategy of the analysis presented in this section, in which we must specify the optimum envelope behavior in the drift space, is not a search for an invariant envelopelike solution, but a matching of the beam envelope from the drift space to the invariant envelope of the booster linac. The model in this case is slightly changed with respect to that shown in Fig. 3: first, the point $z_{c}$ is now located at a variable position given by $z_{c}=[(1+d) / 2+N](\lambda / 2)$, which becomes the end of the rf gun cavity structure, and the beginning of the drift section. Here the quantity $d$ accounts for a change in the first half cell length, i.e., $d=0.25$ indicates a $25 \%$ lengthening $(0.625$ cell). We will also show that, as expected, the optimum field profile for the solenoid magnet is different from the previous (long gun) case, where the magnetic field begins at $z_{B 1}=\lambda / 8$ and ends at $z_{B 2}=(5 / 4$ $+1 / 8) \lambda$. For the compact injector the field start position is shifted downward at $z_{B 1}=\lambda / 2$, while the end position is shifted downward at $z_{B 2}=(7 / 4) \lambda$. As discussed in the following, the longer magnetic field profile is needed to provide more focusing from the solenoid in a case where the ponderomotive $\mathrm{rf}$ focusing in the following cells is not only missing, but the exit defocusing transient kick at the end of the gun must be overcome.

The beam energy $\gamma_{c}$ (at $z=z_{c}$ ) now becomes $\gamma_{c}=\gamma_{2}$ $+(N-1) \pi \alpha$, with

$$
\gamma_{2}=\left[1+\frac{3}{2} \pi \alpha\right]\left[1+\frac{5}{12} d-\left(\frac{5}{2}+\frac{\pi^{2}}{3}\right) \frac{d^{2}}{24}\right]
$$

again the energy at the second iris position (as derived in Appendix A). Since the drift space downstream of $z_{c}$ is free from any accelerating and/or focusing force, the rms envelope equation becomes, in this case,

$$
\sigma^{\prime \prime}-\frac{P}{\sigma}-\frac{\epsilon_{n, \mathrm{th}}^{2}}{\sigma^{3} \gamma_{c}^{2}}=0
$$

where $\sigma^{\prime \prime}=d^{2} \sigma / d z^{2}$ and $P \equiv I / 2 I_{0} \gamma_{c}^{3}$ is now defined as the beam perveance (the assumption $\gamma_{c} \gg 1$ is understood). According to the assumption of quasilaminarity, we neglect the emittance term and cast Eq. (6.1) into the space $(\nu, z)$ as

$$
\nu^{\prime \prime}-1 / \nu=0,
$$

where $\nu(z) \equiv \sigma(z) / \sqrt{P}$. Typical values for the perveance are, in case of a 100 -A beam at $\gamma_{c}=1+5 \pi / 2 \cong 9, P \cong 4 \times 10^{-6}$, so that for a $1-\mathrm{mm}$ beam spot size the quantity $\nu$ is of the order of 1 , as is $\nu^{\prime}$ when $\sigma^{\prime}$ is a few mrad. Equation (6.2) can be derived from a Hamiltonian $H=p_{\nu}^{2} / 2-\ln \nu$ (with $p_{\nu}$ $\equiv \nu^{\prime}$ ), where $H$ is now a constant of the motion, so that

$$
\nu^{\prime}=\sqrt{\nu_{c}^{\prime 2}+2 \ln \left(\nu / \nu_{c}\right)}
$$


which gives the trajectory solution for Eq. (6.2) in integral form:

$$
\int_{1}^{\nu / \nu_{c}} \frac{d x}{\sqrt{\nu_{c}^{\prime 2}+2 \ln x}}=\frac{z-z_{c}}{\nu_{c}} .
$$

This equation actually represents a universally scaled beam spreading curve, representing a universally scaled space-charge beam blowup.

The integral in Eq. (6.4) is not analytically solvable unless the approximation $\left|1-\nu / \nu_{c}\right| \ll 1$ is assumed, which is in fact typical of a rf gun operated in the emittance compensation regime, as the beam size oscillations must be kept small both to prevent nonlaminar trajectories and to keep the oscillation frequency nearly independent of the perveance. Indeed, in practice, the beam exits the gun with a small negative divergence $\sigma_{c}^{\prime}$, so that it is transported up to a space-chargedominated waist with a spot size usually slightly smaller than $\sigma_{c}$. In this case the approximate solution is

$$
\begin{aligned}
\nu(z)= & \nu_{c}\left\{2-\left|\frac{\nu_{c}-\nu_{c}^{\prime} \Delta z}{\nu_{c} \nu_{c}^{\prime}+\Delta z}\right|\right. \\
& \left.\times\left(\frac{1+\nu_{c}^{\prime 2}}{1+\left[\left(\nu_{c}-\nu_{c}^{\prime} \Delta z\right) /\left(\nu_{c} \nu_{c}^{\prime}+\Delta z\right)\right]^{2}}\right)^{1 / 2}\right\},
\end{aligned}
$$

where $\Delta z=z-z_{c}$. It is interesting noticing that Eq. (6.5) actually represents a generalization of a previous result derived by Reiser [18]. The initial conditions $\left(\nu_{c}, \nu_{c}^{\prime}\right)$ are given, in terms of the beam conditions at the gun exit $\left(\tau_{c}, \dot{\tau}_{c}\right)$, by

$$
\nu_{c}=\frac{\tau_{c}}{\gamma^{\prime}}\left(\frac{\gamma_{c}^{3}}{\gamma_{2}}\right)^{1 / 2}, \quad \nu_{c}^{\prime}=\left(\dot{\tau}_{c}+\frac{\tau_{c}}{2}\right)\left(\frac{\gamma_{c}}{\gamma_{2}}\right)^{1 / 2},
$$

recalling that $\left(\nu_{c}, \nu_{c}^{\prime}\right)$ correspond to actual envelope variables, which Eq. (6.6) connects to $\left(\tau_{c}, \dot{\tau}_{c}\right)$, which are secular envelope variables.

In order to match to the invariant envelope at the entrance of the booster linac, we need to find the conditions under which the phase space angle corresponding to the solution to Eq. (6.2) is invariant with respect to the beam current $I$, or, equivalently, to the perveance $P$. This is also equivalent to requiring

$$
\frac{d\left(\sigma^{\prime} / \sigma\right)}{d P}=\frac{d\left(\nu^{\prime} / \nu\right)}{d P}=\frac{1}{\nu^{2}}\left(\nu \frac{d \nu^{\prime}}{d P}-\nu^{\prime} \frac{d \nu}{d P}\right)=0 .
$$

Since $d \nu^{\prime} / d P=\left(1 / \nu^{\prime} \nu\right) d \nu / d P$ [see Eq. (6.3)], we have $d\left(\nu^{\prime} / \nu\right) / d P=\left(1 / \nu^{\prime} \nu^{2}\right)\left(1-\nu^{\prime 2}\right) d \nu / d P$. Therefore, $d\left(\sigma^{\prime} /\right.$ $\sigma) / d P=0$ if either $\nu^{\prime 2}=1$ for all $z$ [which is not possible to fulfill, because it is not a solution of Eq. (6.2)] or $d \nu / d P$
$=0$. Since $\nu$ depends on $P$ through the initial conditions $\left(\nu_{c}, \nu_{c}^{\prime}\right)$, the condition $d \nu / d P=0$ is equivalent to

$$
\frac{d \nu_{c}}{d P}=\frac{d \nu_{c}^{\prime}}{d P}=0,
$$

which in turn is equivalent to the condition

$$
\begin{aligned}
& \frac{d \tau_{c}}{d \Lambda}=0, \\
& \frac{d \dot{\tau}_{c}}{d \Lambda}=0,
\end{aligned}
$$

where the Cauchy current density is given by $\Lambda$ $=I /\left(\gamma^{\prime} \sigma_{r}\right)^{2}$.

It should be noted, however, that the invariance of the phase space angle at the end of the drift given by Eq. (6.7) is achieved only through the invariance of the initial conditions versus the current. The reason for this is that the phase space angle $\nu^{\prime} / \nu$ associated with Eq. (6.5) is not intrinsically invariant, unlike the case of the invariant envelope, where the phase space angle is a constant $\dot{\hat{\tau}} / \hat{\tau}=-1 / 2$. In this respect, Eq. (6.2) does not display any invariant envelope solution, i.e., any solution for which $\nu^{\prime} / \nu=$ const equivalent to the Brillouin flow condition given in Eq. (2.2), where the phase space angle is again a constant $\sigma_{\mathrm{eq}}^{\prime} / \sigma_{\mathrm{eq}}=0$.

To better clarify this point let us examine the equation for small deviations $\delta \nu$ around an equilibrium solution $\nu_{0}$ of Eq. (6.2). Assuming $\delta \nu / \nu_{0} \ll 1$, we find

$$
\delta \nu^{\prime \prime}+\frac{\delta \nu}{\nu_{0}^{2}}=0,
$$

giving stable oscillations with frequency $1 / \nu_{0}$ around the equilibrium solution $\nu_{0}$. As far as the beam envelope can be represented by the approximate solution [Eq. (6.5)] in the drift space, implying that the beam size varies slightly between the initial condition $\nu_{c}$ and the beam spot at the waist $\nu_{w}$, we may identify $\nu_{0}$ roughly with the expression of Eq. (6.5), so that the drift space up to the waist (and slightly further away) is comparable to a quasi-Brillouin flow condition with a local stability condition similar to the one described in Sec. II. Since the beam size, in the absence of any focusing, grows indefinitely after the waist, the frequency of oscillation $1 / \nu_{0}$ around the equilibrium solution is decreasing and the nonlinearities [see Eq. (2.9)] in the oscillations preclude any further vanishing point in the correlated emittance, as clearly illustrated in examples shown below.

It is interesting to notice that Eqs. (6.8) are equivalent to a vanishing correlated emittance at the waist position; this requires that the waist position $\Delta z_{w} \equiv z_{w}-z_{c}$ and the waist beam spot size $\nu_{w}$ are independent of the perveance,

$$
\frac{d \Delta z_{w}}{d P}=0, \quad \frac{d \nu_{w}}{d P}=0,
$$




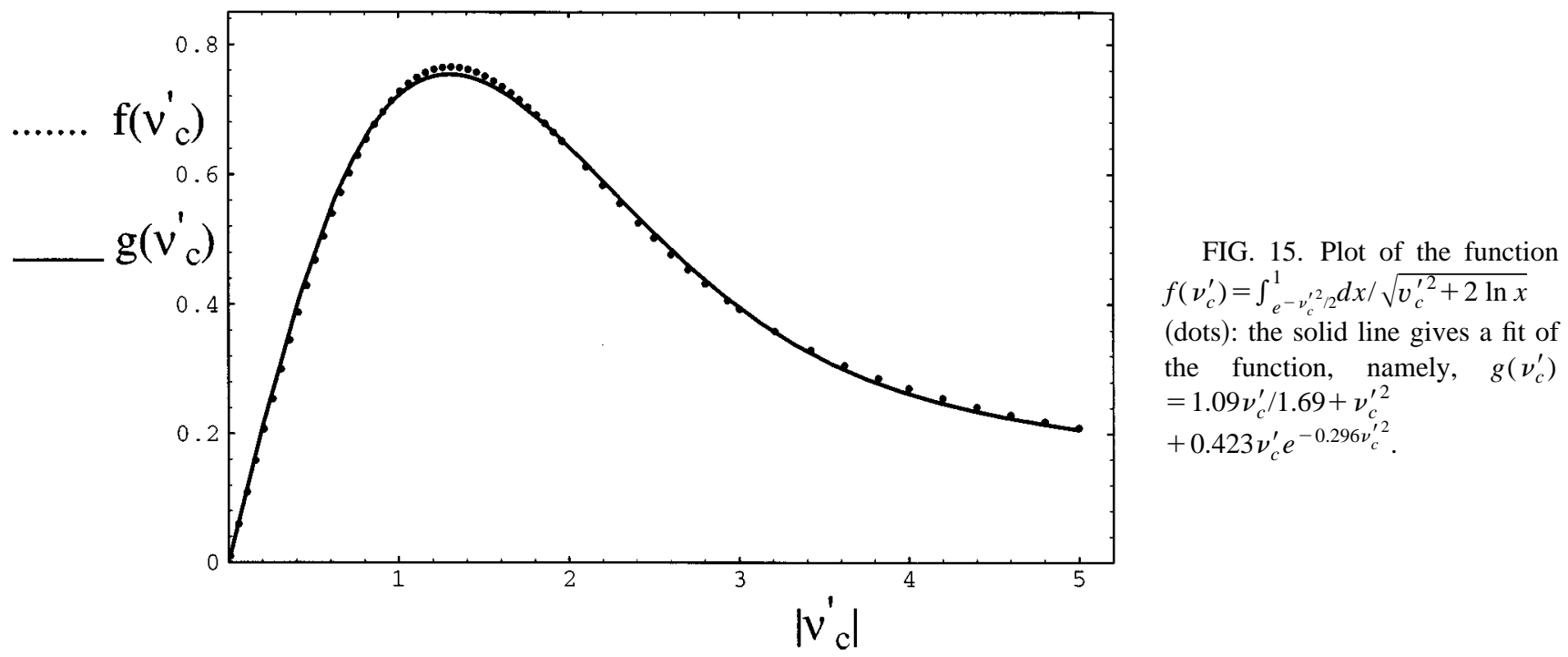

$$
\Delta z_{w}=\nu_{c} f\left(\nu_{c}^{\prime}\right), \quad f\left(\nu_{c}^{\prime}\right) \equiv \int_{e^{-\nu_{c}^{\prime 2} / 2}}^{1} \frac{d x}{\sqrt{\nu_{c}^{\prime 2}+2 \ln x}}, \quad \nu_{w}=\nu_{c} e^{-\nu_{c}^{\prime 2} / 2}
$$

Note the elegant expression for $\nu_{w}$ as a function of $\nu_{c}$ and $\nu_{c}^{\prime}$, which physically indicates that a beam focused too strongly (i.e., $\nu_{c}^{\prime}<-2$ ) will not come to a laminar spacecharge-dominated waist but will likely come to an emittancedominated nonlaminar waist.

The function $f\left(\nu_{c}^{\prime}\right)$ is plotted versus $\nu_{c}^{\prime}$ in Fig. 15. For the purpose of further analysis, we note that $f\left(\nu_{c}^{\prime}\right)$ can be approximated (within a $5 \%$ error) by the function $g\left(\nu_{c}^{\prime}\right)$ $=1.09 \nu_{c}^{\prime} /\left(1.69+\nu_{c}^{\prime 2}\right)+0.423 \nu_{c}^{\prime} e^{-0.296 \nu_{c}^{\prime 2}}$, in the range $\left|\nu_{c}^{\prime}\right|$ $\leqslant 6$. This range easily covers all rf photoinjectors of interest, as a larger value of $\left|\nu_{c}^{\prime}\right|$ implies a strongly convergent beam that will be susceptible to nonlaminar behavior near the waist. The conditions in Eq. (6.10) are now written explicitly in terms of the initial conditions at the start of the drift as

$$
\begin{gathered}
f\left(\nu_{c}^{\prime}\right) \frac{d \nu_{c}}{d P}+\nu_{c} \frac{d f\left(\nu_{c}^{\prime}\right)}{d \nu_{c}^{\prime}} \frac{d \nu_{c}^{\prime}}{d P}=0, \\
\frac{d \nu_{c}}{d P}-\nu_{c} \nu_{c}^{\prime} \frac{d \nu_{c}^{\prime}}{d P}=0 .
\end{gathered}
$$

This system of equations allows solutions different from $d \nu_{c} / d P=d \nu_{c}^{\prime} / d P=0$ if the determinant of the coefficient matrix, det $M=-\nu_{c}\left[\nu_{c}^{\prime} f\left(\nu_{c}^{\prime}\right)+d f\left(\nu_{c}^{\prime}\right) / d \nu_{c}^{\prime}\right]$, is vanishing. By applying the Leibniz formula for the derivation of definite integrals, we find $d f\left(\nu_{c}^{\prime}\right) / d \nu_{c}^{\prime}=1-\nu_{c}^{\prime} f\left(\nu_{c}^{\prime}\right)$, so that $\operatorname{det} M$ $=-\nu_{c}$, which can never be vanishing, implying that the conditions $d \nu_{c} / d P=d \nu_{c}^{\prime} / d P=0$ and $\left[d \Delta z_{w} / d P=0 ; d \nu_{w} / d P\right.$ $=0]$ are in fact equivalent.

In order to derive the solution to Eq. (6.8) in terms of the beam conditions $\left(\tau_{2}, \dot{\tau}_{2}\right)$ at the second iris position $(z$ $=z_{2}$ ), as reported in Appendix A, we approximate the envelope equation in the region of drift with applied solenoid field (i.e., $z_{2}<z<z_{B 2}$ ) as

$$
\nu^{\prime \prime}+K_{r} \nu=1 / \nu_{2},
$$

where $\nu_{2} \equiv \sigma_{2} / \sqrt{I /\left(2 I_{0} \gamma_{2}^{3}\right)}$, and $K_{r}=\left(b \gamma^{\prime} / \gamma_{2}\right)^{2}$, and so the space-charge term is taken as a constant, its value assumed at the gun exit. This is valid at the present point only for a 1 $+1 / 2$ cell gun, for which the beam energy at the exit, $\gamma_{2}$, is constant all over the drift space: however, the treatment can be easily generalized to the case of a $N+1 / 2(1+d)$ cell gun. In the $N=1$ the drift space is divided into two parts. In the first one, from $z=z_{2}=(3 / 4) \lambda$ up to $z=z_{c}=z_{B 2}=(7 / 4) \lambda$, the beam is subject to a focusing solenoid field, while for $z$ $>z_{c}$ the drift is in free space. Under this approximation Eq. (6.12) can be easily solved to find

$$
\begin{gathered}
\nu_{c}=\nu_{2} \cos \theta+\frac{(1-\cos \theta)}{\nu_{2} K_{r}}+\nu_{2}^{\prime} \frac{\sin \theta}{\sqrt{K_{r}}}, \\
\nu_{c}^{\prime}=-\nu_{2} \sqrt{K_{r}} \sin \theta+\frac{\sin \theta}{\nu_{2} \sqrt{K_{r}}}+\nu_{2}^{\prime} \cos \theta,
\end{gathered}
$$

where $\theta=\sqrt{K_{r}}\left(z-z_{2}\right)$, and hence

$$
\begin{gathered}
\frac{d \nu_{c}}{d P}=\frac{d \nu_{2}}{d P}\left[\cos \theta-\frac{(1-\cos \theta)}{\nu_{2}^{2} K_{r}}\right]+\frac{d \nu_{2}^{\prime}}{d P} \frac{\sin \theta}{\sqrt{K_{r}}} \\
\frac{d \nu_{c}^{\prime}}{d P}=\frac{d \nu_{2}}{d P}\left[-\sqrt{K_{r}} \sin \theta-\frac{\sin \theta}{\nu_{2}^{2} \sqrt{K_{r}}}\right]+\frac{d \nu_{2}^{\prime}}{d P} \cos \theta
\end{gathered}
$$




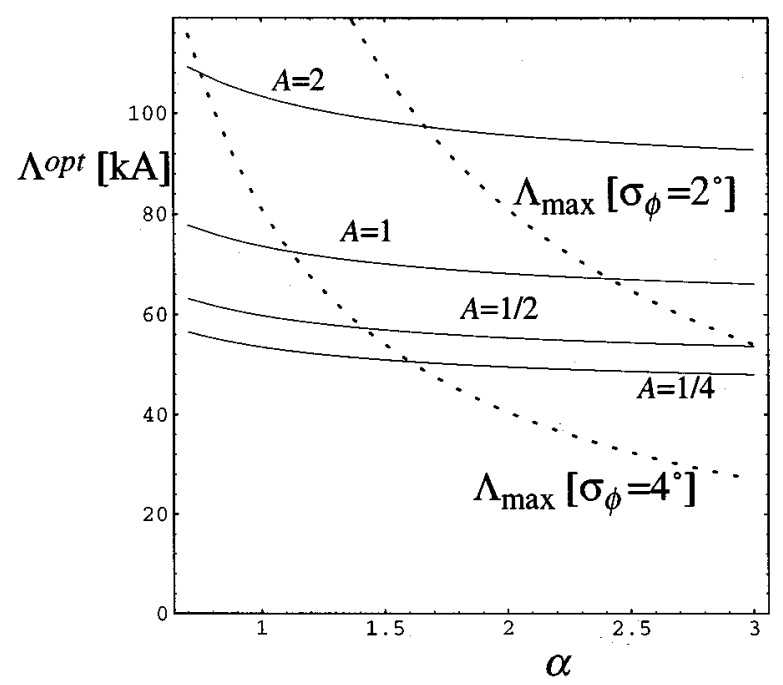

FIG. 16. Operating diagram in the $(\alpha, A)$ plane for a short (1 $+1 / 2$ cell) photoinjector.

The determinant of the coefficient matrix is derived to be $\operatorname{det} M_{2}=1+(1-\cos \theta) / \nu_{2}^{2} K_{r}$, which clearly implies $\operatorname{det} M_{2}$ $>1$, indicating that the only solution is $d \nu_{2} / d P=d \nu_{2}^{\prime} / d P$ $=0$, which we know to be equivalent to

$$
\frac{d \tau_{2}}{d \Lambda}=0, \quad \frac{d \dot{\tau}_{2}}{d \Lambda}=0,
$$

where $\tau_{2}$ and $\dot{\tau}_{2}$ are specified in Eqs. (A7) and (A8) as functions of $(\alpha, A, \Lambda, b)$. Following the same procedure as in Sec. V, we solve these two equations by expressing their roots as $\Lambda=\Lambda(\alpha, A)$ and $b=b(\alpha, A)$. The system is highly nonlinear, so that we start by expressing the first part of Eqs. (6.15) as

$$
\frac{d \tau_{2}}{d \Lambda}=\frac{1}{\Lambda}\left\{\left(\frac{\Lambda 2 \gamma_{2}}{I_{0}}\right)^{1 / 2} \frac{\pi \zeta(A)}{4}\left[1-\frac{\ln \gamma_{2}}{\gamma_{2}-1}\right]-\tau_{2} / 2\right\}=0,
$$

which can be solved for the variable

$$
b=\sqrt{2}\left\{1+\left(1-\frac{\pi \zeta(A) \Lambda}{4 I_{0}}\right)\left[1-\frac{\ln \gamma_{2}}{\gamma_{2}-1}\right]\right\}^{1 / 2} / \ln \left(\frac{\gamma_{2}}{\gamma_{b}}\right) .
$$

Substituting back into the second part of Eqs. (6.15), we obtain an equation in $\Lambda, \alpha$, and $A$. By a fitting procedure we obtain the following solutions:

$$
\begin{aligned}
\Lambda^{\mathrm{opt}}[\mathrm{kA}]= & 57.3-12.4 \alpha+2.63 \alpha^{2}+26.2 A-1.78 \alpha A \\
& +1.86 A^{2}
\end{aligned}
$$

and

$$
b^{\mathrm{opt}}=1.49+\frac{1.67}{\sqrt{\alpha}}-\frac{2.07}{\alpha^{1 / 4}}
$$

The Cauchy perveance $\Lambda^{\mathrm{opt}}$ is plotted in Fig. 16 (solid lines) as a function of $\alpha$ for some usual values of $A$. It is interesting to note that $\Lambda^{\text {opt }}$ is nearly independent of $\alpha$ for $\alpha \geqslant 1.5$, while it decreases almost linearly with $\alpha$ for $\alpha$

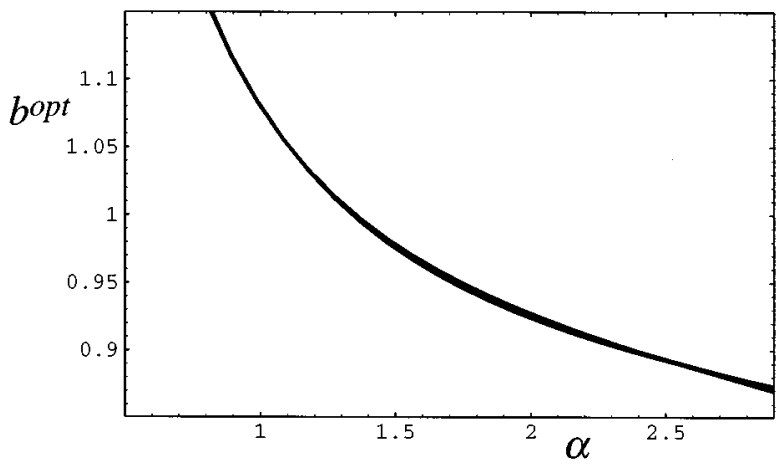

FIG. 17. Parameter $b^{\text {opt }}$, plotted as a function of $\alpha$, at some values of the bunch aspect ratio $A$.

$\leqslant 1.5$. Its dependence on $A$ is fairly linear in most of the diagram, so that the following scaling laws hold:

$$
\Lambda^{\mathrm{opt}} \propto\left\{\begin{array}{l}
A \quad \text { if } \alpha \geqslant 1.5, \\
A(3-\alpha) \quad \text { if } \alpha \leqslant 1.5,
\end{array}\right.
$$

which can be cast in terms of the bunch charge $Q_{b}$, cathode spot size $\sigma_{r}$, accelerating gradient $\gamma^{\prime}$, and rf wave number $k$,

$$
Q_{b} \propto\left\{\begin{array}{l}
\sigma_{r}^{3} \gamma^{\prime 2} \text { if } \alpha \geqslant 1.5 \\
\sigma_{r}^{3} \gamma^{\prime 2}\left(3-\gamma^{\prime} / k\right) \quad \text { if } \alpha \leqslant 1.5
\end{array}\right.
$$

which resemble the scaling laws reported in Ref. [17].

As in Fig. 6, the Cauchy perveance $\Lambda_{\max }$ [see Eq. (5.5)] compatible with the maximum charge limit is plotted in Fig. 16 (dotted lines) for a bunch length $\sigma_{\phi}=2^{\circ}$ (the higher line) and $\sigma_{\phi}=4^{\circ}$ (the lower line). The parameter $b^{\text {opt }}$, which represents the ratio between magnetic and rf focusing, turns out to be nearly independent of the aspect ratio $A$, as shown in Fig. 17, where $b^{\text {opt }}$ is plotted as a function of $\alpha$ for different values of $A$ [it should be noted that Eq. (6.18) displays a simplified form for $b^{\text {opt }}$, which has already removed the very weak dependence on $A]$. It should be also noted that $b^{\text {opt }}$ is much higher than the analogous parameter $b_{s}$ (see Fig. 7) required in the case of the indefinitely long photoinjector. This is due to the facts that, not only is the additional rf focusing applied in the long photoinjector missing here, but the transient defocusing at the end of the gun occurs at a large beam spot size; these effects create the need for enhanced focusing from the external solenoid.

The current density $J$ corresponding to the $\Lambda_{s}$ lines is plotted (solid lines) in Fig. 18, for some selected rf frequencies $(0.65,1.3,3$, and $6 \mathrm{GHz})$, at aspect ratio $A=1$. The maximum limits for the current density are also reported, in the case of $\sigma_{l}=5 \mathrm{psec}$ (higher dashed line) and $\sigma_{t}$ $=10 \mathrm{psec}$ (lower dashed line). The required cathode spot size $\sigma_{r}$ (for the case of a 1-nC bunch charge) are plotted in Fig. 19, for the same set of frequencies and at different bunch aspect ratios.

It should be noted that the operating point to achieve the emittance compensation shown in Figs. 14(a) and 14(b) has been derived by the operating diagram in Fig. 16, selecting the point $\alpha=1.8, A=1 / 2, \Lambda=56 \mathrm{kA}$, giving at the $L$ band $E_{0}=50 \mathrm{MV} / \mathrm{m}, \quad \sigma_{r}=0.76 \mathrm{~mm}$, and $I=78 \mathrm{~A}, I^{+}=97 \mathrm{~A}$, 


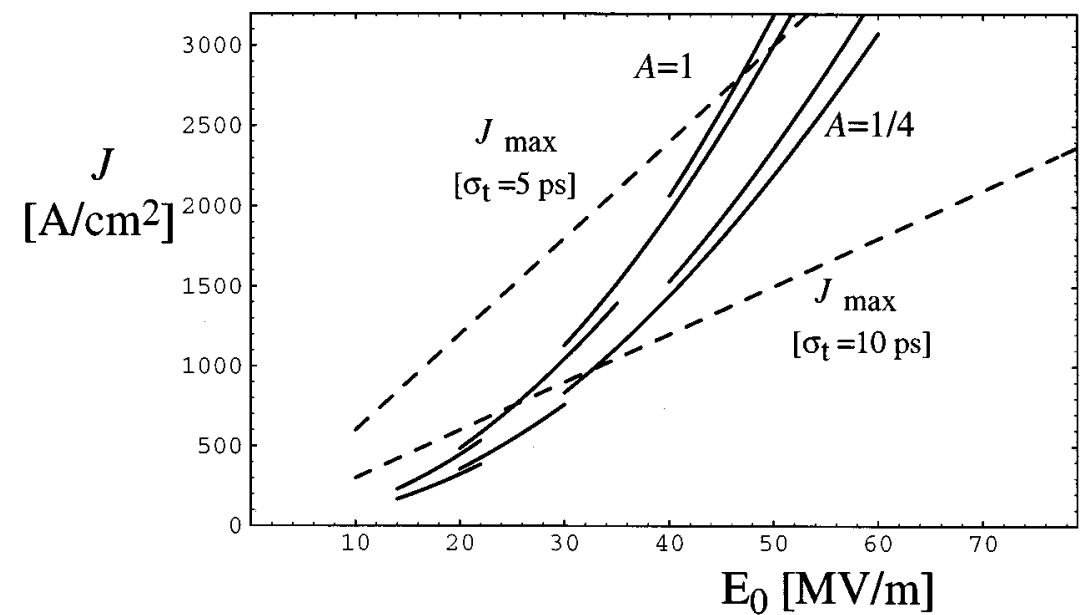

FIG. 18. Current density $J$ plotted as a function of the cathode peak field $E_{0}[\mathrm{MV} / \mathrm{m}]$, at different $\mathrm{rf}$ frequencies, for a short photoinjector operated in the emittance correction regime.

$I^{-}=53 \mathrm{~A}$ (for a 1-nC bunch charge). The magnetic field parameter is $b^{\mathrm{opt}}=0.94$, corresponding to $B_{0}=1.57 \mathrm{kG}$.

We have already solved exactly for the beam waist size $\nu_{w}=\nu_{c} e^{-\nu_{c}^{\prime 2} / 2}$, but have not explicitly given an expression for the position of the space-charge-dominated waist when the gun is operated under the conditions specified by Eqs. (6.17) and (6.18), as required to achieve emittance correction. We restrict the discussion here to the case of $N=1$, but the results are easily extendible to $N \geqslant 2$. At the gun exit $(z$ $\left.=z_{2}\right)$ we have [cf. Eq. (6.6)] $\nu_{2}^{\mathrm{opt}}=\left(\tau_{2}^{\mathrm{opt}} / \gamma^{\prime}\right) \gamma_{2}, \nu_{2}^{\prime \text { opt }}$ $=\left(\dot{\tau}_{2}^{\text {opt }}+\tau_{2}^{\text {opt }} / 2\right)$, where the superscript opt indicates that we have substituted Eqs. (6.17) and (6.18) into (A7) and (A8), so that $\tau_{2}^{\text {opt }}, \dot{\tau}_{2}^{\text {opt }}, \nu_{2}^{\text {opt }}$ are now functions of only $\alpha$ and $A$, while $\nu_{2}^{\text {opt }}$ can be represented by a function of $\alpha$ and $A$ divided by $\gamma^{\prime}$, e.g., $\nu_{2}^{\text {opt }} \equiv f_{\nu}(\alpha, A) / \gamma^{\prime}$. Substituting $\nu_{2}^{\text {opt }}$, $\nu_{2}^{\prime \text { opt }}$ for $\nu_{2}, \nu_{2}^{\prime}$ in Eq. (6.13), we find

$$
\begin{gathered}
\nu_{c}^{\mathrm{opt}}=\frac{1}{\gamma^{\prime}}\left[f_{\nu} \cos \theta^{\mathrm{opt}}+\frac{\left(1-\cos \theta^{\mathrm{opt}}\right)}{f_{\nu}\left(b^{\mathrm{opt}}\right)^{2}} \gamma_{2}^{2}+\nu_{2}^{\prime \mathrm{opt}} \frac{\sin \theta^{\mathrm{opt}}}{b^{\mathrm{opt}}} \gamma_{2}\right], \\
\nu_{c}^{\text {opt }}=-f_{\nu} \sin \theta^{\mathrm{opt} /} \gamma_{2}+\frac{\sin \theta^{\mathrm{opt}}}{f_{\nu} b^{\mathrm{opt}}} \gamma_{2}+\nu_{2}^{\prime \mathrm{opt}} \cos \theta^{\mathrm{opt}}
\end{gathered}
$$

where, again, $\nu_{c}^{\prime \text { opt }}$ is a function of only $\alpha$ and $A$, and $\nu_{c}^{\text {opt }}$ can be represented by a function of $\alpha$ and $A$ divided by $\gamma^{\prime}$, since the term inside square brackets is a function of only $\alpha$ and $A\left[\theta^{\mathrm{opt}}=b^{\mathrm{opt}}\left(\gamma_{c}-\gamma_{2}\right) / \gamma_{2}\right]$.

Now employing Eq. (6.10) we finally find the waist position $z_{w}^{\text {opt }}$ and spot size $\sigma_{w}^{\text {opt }}$ under optimum operating conditions,

$$
\begin{aligned}
& z_{w}^{\mathrm{opt}}=\frac{7}{4} \lambda+\frac{1}{\gamma^{\prime}}\left[10.8 \alpha+1.48 \alpha^{2}-1.18 A-1.07 \alpha A-3\right] \\
& \sigma_{w}^{\mathrm{opt}}[\mathrm{mm}]= \frac{\sqrt{I[\mathrm{~A}]}}{\gamma^{\prime}\left[\mathrm{m}^{-1}\right]}\left[3.76+1.56 \alpha-1.58 \alpha^{2}+0.26 \alpha^{3}\right. \\
&\left.+0.56 A+0.914 \alpha A-0.11 \alpha^{2} A-0.15 \alpha A^{2}\right] .
\end{aligned}
$$

As an example, for an $L$-band injector operated at $\nu_{\mathrm{rf}}$ $=1.3 \mathrm{GHz}$, with $E_{0}=56 \mathrm{MV} / \mathrm{m}\left(\gamma^{\prime}=55 \mathrm{~m}^{-1}\right.$, and $\left.\alpha=2\right)$ the optimum $\Lambda$ at $A=1$ will be $\Lambda^{\text {opt }}=68 \mathrm{kA}$, while $b^{\text {opt }}$ $=0.92$, so that the solenoid field will be $B_{0}=1.7 \mathrm{kG}$. Assuming a bunch charge $Q_{b}=1 \mathrm{nC}$, the laser spot size at the cathode $\sigma_{r}$ will be, recalling that $\sigma_{r}$ $=\sqrt[3]{(c / \sqrt{2 \pi}) Q_{b} A / \Lambda \gamma^{\prime 2}}, \quad \sigma_{r}=0.84 \mathrm{~mm}$. The beam current will be given by $I=Q_{b} c / \sqrt{2 \pi} \sigma_{z}=Q_{b} c A / \sqrt{2 \pi} \sigma_{r}=142 \mathrm{~A}$, so that the beam spot size at the waist will be $\sigma_{w}^{\text {opt }}$ $=0.94 \mathrm{~mm}$, while at the gun exit $\sigma_{2}=1.9 \mathrm{~mm}$. The waist

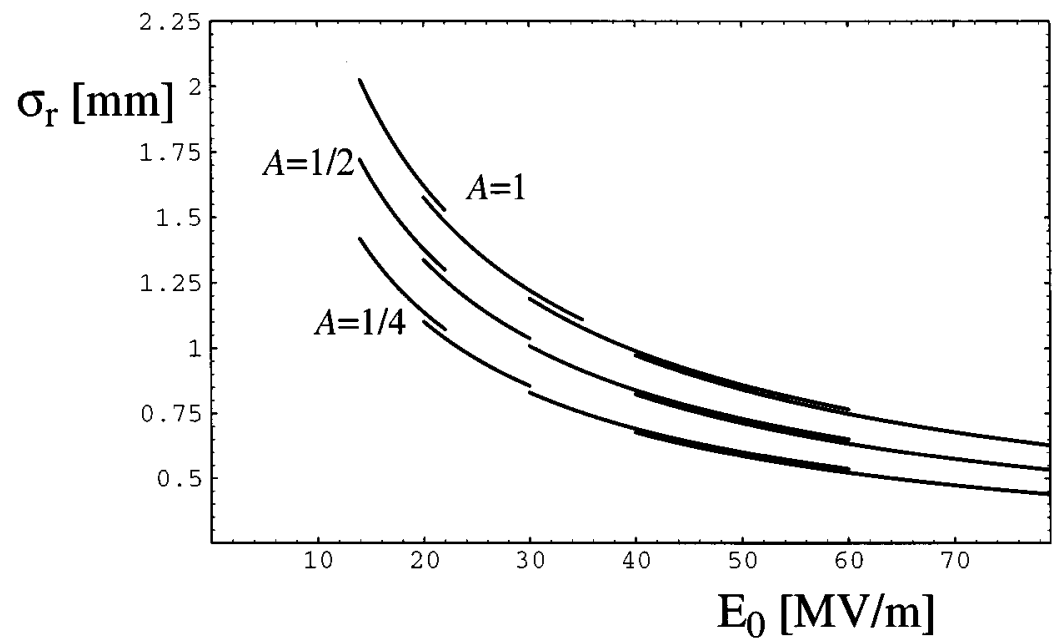

FIG. 19. Cathode spot size $\sigma_{r}$, plotted vs the cathode peak field $E_{0}[\mathrm{MV} / \mathrm{m}]$, at different $\mathrm{rf}$ frequencies, for a short photoinjector operated in the emittance correction regime. 
TABLE I. Properties of invariant envelope flow, where possible, in various types of beam transport and acceleration.

\begin{tabular}{|c|c|c|c|}
\hline Beam transport & Invariant envelope & Space & Emittance damping \\
\hline Standing wave linac & $\begin{array}{c}\text { Secular } \\
\frac{2 e^{-y / 2}}{\sqrt{\eta / 2 \sin ^{2}\langle\phi\rangle+1}}\end{array}$ & $\begin{array}{c}\text { Cauchy } \\
\text { dimensionless }\end{array}$ & Yes \\
\hline $\begin{array}{l}\text { Standing wave linac } \\
\text { plus solenoid }\end{array}$ & $\begin{array}{c}\text { Secular } \\
\frac{e^{-y / 2}}{\sqrt{\left(\eta / 8+b^{2}\right) / \sin ^{2}\langle\phi\rangle+\frac{1}{4}}}\end{array}$ & $\begin{array}{c}\text { Cauchy } \\
\text { dimensionless }\end{array}$ & Yes \\
\hline $\begin{array}{l}\text { Traveling wave } \\
\text { linac }\end{array}$ & $\begin{array}{l}\text { Actual } \\
2 e^{-y / 2}\end{array}$ & $\begin{array}{c}\text { Cauchy } \\
\text { dimensionless }\end{array}$ & Yes \\
\hline $\begin{array}{l}\text { Traveling wave } \\
\text { linac plus solenoid }\end{array}$ & $\begin{array}{l}\text { Actual } \\
e^{-y / 2}\end{array}$ & $\begin{array}{c}\text { Cauchy } \\
\text { dimensionless }\end{array}$ & Yes \\
\hline & $\sqrt{b^{2} / \sin ^{2}\langle\phi\rangle+\frac{1}{4}}$ & & \\
\hline Drift & No & Real dimensionless & No \\
\hline Drift plus solenoid & $\begin{array}{l}\text { Brillouin Flow } \\
\sigma_{e q}=\sqrt{P / K_{r}}\end{array}$ & Real dimensionless & No \\
\hline
\end{tabular}

will be located at $z_{w}^{\mathrm{opt}}=0.793 \mathrm{~m}$. It is remarkable to note that the waist position scales with explicit dependence only on the rf field and wavelength, not the bunch charge and/or current (which are derived quantities), in agreement with what is observed in Ref. [17].

For the case where the first half cell is lengthened by $20 \%$, as is done in many new rf gun designs, we have recalculated $\Lambda^{\text {opt }}$ and $b^{\text {opt }}$ to obtain

$$
\begin{aligned}
\Lambda^{\mathrm{opt}}[\mathrm{kA}]= & 58.33-11.83 \alpha+2.52 \alpha^{2}+27.34 A-1.816 \alpha A \\
& +1.88 A^{2}
\end{aligned}
$$

and

$$
b^{\mathrm{opt}}=1.38+\frac{1.52}{\sqrt{\alpha}}-\frac{1.86}{\alpha^{1 / 4}},
$$

i.e., behavior very close to that of the standard half cell. The predicted position and spot of the waist now become

$$
\begin{array}{r}
z_{w}^{\mathrm{opt}}=\frac{7}{4} \lambda+\frac{1}{\gamma^{\prime}}\left[9.94 \alpha+2.59 \alpha^{2}+0.18 A-2.65 \alpha A-2.96\right], \\
\sigma_{w}^{\mathrm{opt}}[\mathrm{mm}]=\frac{\sqrt{I[\mathrm{~A}]}}{\gamma^{\prime}\left[\mathrm{m}^{-1}\right]}\left[5.2+2.14 \alpha-1.77 \alpha^{2}+0.27 \alpha^{3}\right. \\
\left.+0.19 A+0.95 \alpha A-0.058 \alpha^{2} A-0.19 \alpha A^{2}\right] .
\end{array}
$$

Taking the same example as before, i.e., for an injector operated at the $L$ band $\left(\nu_{\mathrm{rf}}=1.3 \mathrm{GHz}\right)$ and $E_{0}$ $=55.7 \mathrm{MV} / \mathrm{m}$ (hence $\gamma^{\prime}=54.6 \mathrm{~m}^{-1}$ and $\alpha=2$ ) the optimum $\Lambda$, at $A=1$, is now $\Lambda^{\mathrm{opt}}=70.4 \mathrm{kA}$, while $b^{\mathrm{opt}}=0.89$, so that the solenoid field will be $B_{0}=1.64 \mathrm{kG}$. The relaxation of the magnetic field required reflects the additional rf focusing that the elongated cell provides. With a bunch charge $Q_{b}$ $=1 \mathrm{nC}$, the laser spot size at the cathode $\sigma_{r}$ is now $\sigma_{r}$
$=0.83 \mathrm{~mm}$, and the beam current becomes $I=144 \mathrm{~A}$, so that the main parameters are almost unchanged, as is the waist position, which is $z_{w}^{\mathrm{opt}}=0.804 \mathrm{~m}$. On the other hand, since the beam does not expand as much before solenoid focusing, the beam spot size at the waist is now larger, being $\sigma_{w}^{\text {opt }}$ $=1.3 \mathrm{~mm}$.

The behavior of the emittance evolution after the injection of the beam into the booster linac is closely related to that discussed at the end of Sec. V concerning the case of a long injector. In the present case, however, the beam has been already compensated at the entrance of the linac, in the sense that the beam has undergone a full envelope oscillation, and has achieved a local minimum in both emittance and envelope. Therefore the emittance will again tend to rise, but the damping effects of operation near the invariant envelope hold this rise in check. A further diminishing of the emittance is expected after an additional perturbed beam envelope (plasma) period, as is illustrated in Fig. 14(a). This "double" compensation shows the power of the split gunbooster linac configuration.

\section{CONCLUSIONS}

We have discussed, in some detail, the properties of the invariant envelope, which is a particular beam propagation mode characterized by a phase space angle that is a global constant. Under the hypothesis of quasilaminarity, which is equivalent to the assumption that the beam is space-charge dominated and the number of plasma oscillations considered are small (in order to avoid transverse and longitudinal mixing), we have shown that the invariant envelope is a propagation mode that damps the correlated emittance-provides emittance compensation-so that the possible emittance dilution of a beam, due to longitudinal-transverse correlations caused by either space charge or any other source (rf, etc.), can be corrected by transporting the beam under an invariant envelope mode. While we have concentrated here on standing wave linacs, the invariant envelope exists in other types 
of structures, with and without externally applied focusing forces, as summarized in Table I.

In general, rf linacs allow acceleration under the invariant envelope both in standing and traveling wave operation where the correlated emittance oscillations are damped due to acceleration, as on the invariant envelope the beam spot, and eventual compensated emittance diminishes as $\gamma^{-1 / 2}$. For completeness, we have shown in the table the invariant envelope associated with the cases of both traveling and standing wave linacs with additional uniform solenoidal focusing. Traveling wave linacs operated with an extra magnetic focusing may be in principle equivalent to standing wave linacs, where the focusing is provided by the rf ponderomotive effect, as long as the magnetic focusing ratio $b$ $\equiv c B_{0} / E_{0}$ is chosen to have the value $b=\sqrt{\eta / 2}$.

Drift spaces, on the other hand can be operated in the invariant envelope only with an external focusing to set up a true Brillouin flow condition: the motion in the drift space after a compact photoinjector is, in this respect, only an approximation of the invariant envelope. In any event, spacecharge-driven emittance oscillations are not damped in drifts, so that one must quickly accelerate the beam after the drift, starting from the first emittance minimum, in order to avoid the onset of wave-breaking processes (i.e., nonlaminar effects due to nonlinearities), which transform the reversible emittance oscillation into an irreversible, thermal-like emittance growth as discussed in Ref. [19].

A transport line made by different sections that are all operated under their own invariant envelope mode is of course a globally invariant envelope beam propagation. The final design of a photoinjector that is be operated in the ideal emittance correction regime will be therefore made up by an interlocked sequence of accelerating and drift sections properly matched and operated under invariant envelopes. Care must be taken that this array includes the final transport from the booster linac to the application, so as to avoid emittance growth after initial compensation: this is of course true as far as the beam is still space-charge dominated in the sense discussed above in this paper. In general this means, for transport that is longer than one-quarter of a plasma wavelength, that the beam be focused often enough (typically by quadrupoles) to approximate Brillouin flow after the photoinjector linac, with the beam controlled so as to not make large excursions in spot size. For discrete focusing elements such as quadrupoles, this means that the elements must be placed within one-quarter of a plasma wavelength of each other.

In matching different sections one should, however, be careful about what kind of orbit the invariant envelope is expressed as: secular or actual. In standing wave linacs the envelope is given in terms of a secular orbit, i.e., an orbit averaged over the cell-to-cell oscillations, so that at the entrance of the structure one must subtract a focusing kick of strength $\Delta \sigma^{\prime}=-\gamma^{\prime} \sigma / 2 \gamma$ to the beam envelope conditions of the previous section in order to match the secular envelope. In the case the previous section is the drift space between the short rf gun and a booster linac, one should position the space-charge-dominated waist directly at the entrance of the linac, as discussed in Sec. VI: the initial divergence of the secular envelope in the booster will be in this way $\sigma^{\prime}=-\gamma^{\prime} \sigma / 2 \gamma$, which is exactly the first condition on the invariant envelope. The second condition, i.e., $\sigma$ $=\left(2 / \gamma^{\prime}\right) \sqrt{I / 3 I_{0} \gamma}$, can be easily achieved by simply tuning the accelerating gradient $\gamma^{\prime}$ for a given energy, current, and spot size of the beam at the booster entrance. It is remarkable to note that this prescription on the matching condition has been observed in several simulations of rf photoinjectors [20].

If the booster linac is as well approximated as the pure traveling wave structure the matching conditions may seem different because the invariant envelope is expressed in terms of the actual physical orbit $\sigma=\left(2 / \gamma^{\prime}\right) \sqrt{I / 2 I_{0} \gamma}$. It should be noted, however, that this orbit still has the same associated phase space angle $\sigma^{\prime}=-\gamma^{\prime} \sigma / 2 \gamma$, and that physical transient kick applied at the booster entrance is identical to that applied in the standing wave case. Thus a parallel beam should still be injected into a traveling wave booster in order to match the invariant envelope conditions, as is again in agreement with the results of multiparticle simulations [21]. It can therefore be seen that the natural matching of a parallel beam from a drift to the invariant envelope in an accelerating section is in fact due to the fortuitous relationship between adiabatic damping in trace space and the transient kick the particles feel as they enter the accelerator. This relationship can be explained by a Hamiltonian approach to the dynamics; the radial entrance kick is set by the need to conserve canonical momentum. As the particle enters the accelerator, it must pick up a radial mechanical momentum opposite to the radial field momentum, which is proportional to $\gamma^{\prime}$, a condition that guarantees that a generalized Brillouin equilibrium (invariant envelope) can be obtained.

As a final related example, in the case of a long multicell rf photoinjector structure we recall that the beam, if transported under the invariant envelope, must also leave the photoinjector cavity with zero divergence. Due to the typical high energy of the beam, as in the case of the $10+1 / 2$ cell AFEL photoinjector [22], the beam envelope is assumed to stay parallel for a long drift after leaving the photoinjector [23]. Therefore, a parallel matched beam emerging from a long multicell photoinjector is therefore a sign of proper operation in the emittance compensation regime, as experimentally observed [24]. To restate the conclusions of the previous paragraphs, parallel beams, which are the analogue of the invariant envelope in a drift, match invariant envelopes upon both entrance and exit to accelerating sections.

We have pointed out that most emittance compensated photoinjectors are operated near the optimum conditions predicted by the theory presented in this paper. These conditions, in which the beam is nearly matched to generalized equilibria, have been discussed in detail in this work, and prescriptions for obtaining them have been given for many cases of interest. These prescriptions, and the general physical reasoning behind their generation, can therefore be considered to be guides for optimized performance of spacecharge-dominated beams undergoing acceleration, focusing, and drifts.

In the future, this work will be extended to allow an estimate of the residual emittance of a beam after compensation. The most readily identifiable sources of residual emittance are thermal cathode effects, radial nonlinearities, amplitude dependence of beam oscillation frequencies, and beam phase space bifurcations. The effects of radial nonlinearities (within a slice) under laminar conditions are not problematic, 
since the radial electric field will depend only on the beam size and the enclosed current. Under these conditions, the envelope approach can be easily extended to show that this source of emittance behaves exactly as the linear longitudinally correlated emittance discussed so far in this paper. Likewise, amplitude-dependent frequency effects, anharmonicity of different slice motion, which do give rise to a residual correlated emittance due to dynamical nonlinearity, are treatable within the context of a laminar beam analysis.

On the other hand, thermal cathode effects and phase space bifurcations are beyond the reach of laminar beam analysis, by definition. Because cathode effects are truly stochastic and not dependent on beam dynamics, they therefore can be considered to be an uncorrelated source of emittance, which can neither grow nor diminish. This source of residual emittance must be determined by experimental study of the photoelectric emission process. The subject of phase space bifurcations is perhaps the most challenging from the analytical point of view. The bifurcation in this case is due to beam slices that have "crossover" waists, in which the electrons cross the axis, and the minimum beam size is determined by the slice emittance. This type of event is termed wave breaking in phase space, and represents a complete local violation of the laminarity condition. When bifurcations are typically encountered, one enters a hybrid regime where the beam tails are emittance dominated while the beam core is still in laminar space-charge flow. While it is not clear how to deal with this case within the context of the present approach, it is clear that this effect is a major contributor to emittance growth in rf photoinjector beams, and must therefore be seriously explored.

In addition to these extensions to cylindrically symmetric beam analysis, the generalization of this analysis to highly asymmetric beam $\left(\sigma_{x} \gg \sigma_{y}\right)$ and emittance $\epsilon_{x} \gg \epsilon_{y}$ photoinjectors $[20,25]$ is straightforward. This subject which is of high interest for linear collider electron source applications, is planned to be explored in a forthcoming investigation.

\section{ACKNOWLEDGMENTS}

The authors would like to thank B. Carlsten, E. Colby, K.J. Kim, P. O'Shea, D. Palmer, and R. Sheffield for useful discussions and comments. This work was supported in part by U.S. Department of Energy Grants DE-FG03-93ER40796 and DE-FG03-92ER40693, the Alfred P. Sloan Foundation Grant BR-3225, and the Instituto Nazionale di Fisica Nucleare.

\section{APPENDIX A}

The expressions for the beam exit conditions $\sigma_{2}$ and $\sigma_{2}^{\prime}$ at the second iris location $z=z_{2}$ are reported in this Appendix. The expressions given have been derived for a Gaussian charge distribution in the bunch, of dimensions $\sigma_{r}$ and $\sigma_{z}$, with the range of validity specified by $\alpha>1 / 2$ and $Q_{b}[\mathrm{nC}]$ $<E_{0}[\mathrm{MV} / \mathrm{m}] / 10$, as extensively discussed elsewhere [7]. The formulas reported here correspond to the particular case $\phi=\pi / 2$ examined in Ref. [7], augmented with the focusing effects of the solenoidal magnetic field.

Let us define the beam energy $\gamma_{1}$ and $\gamma_{2}$ at the first and second iris locations, namely, $\gamma_{1}=1+\pi \alpha / 2$ and $\gamma_{2}=1$
$+3 \pi \alpha / 2$. The solenoid field starts at $z_{b}=\lambda / 8$ and extends to $z_{c}=(1 / 8+5 / 4) \lambda$, and the transverse forces imparted to beam electrons during acceleration are represented by a defocusing rf term $\Delta p^{\text {rf }}$, a defocusing space-charge term $\Delta_{\mathrm{SC}}$, and a focusing term $\Delta_{B}$ produced by the magnetic field of the solenoid. Expressed in terms of two auxiliary quantities, $\mu$ $=\Sigma_{1}^{\infty} a_{n}(\mu=1$ for an ideal first harmonic field for which $\left.a_{1}=1, a_{3}=a_{5}=\cdots=0\right)$ and $\mu_{\mathrm{SC}}=\pi I \zeta(A) / 4 I_{0} \gamma^{\prime 2} \sigma_{r}^{2}$, they take the form

$$
\begin{gathered}
\Delta p^{\mathrm{rf}}=\mu\left[1+\mu-\frac{\mu \ln \left(\gamma_{1}\right)}{\gamma_{1}-1}\right]-\frac{\eta\left(1-(\alpha / 4 !)^{2}\right) \ln \left(\gamma_{2}\right)}{8} \\
\times\left[1+\mu-\frac{\mu \ln \left(\gamma_{1} \sqrt[3]{\left.\gamma_{2}\right)}\right.}{2\left(\gamma_{1}-1\right)}\right] \\
\Delta_{\mathrm{SC}}=\left(\mu+\mu_{\mathrm{SC}}\right)\left[1-\frac{\ln \left(\gamma_{2}\right)}{\gamma_{2}-1}\right] \\
\Delta_{B}=b^{2} \ln ^{2}\left(\gamma_{2} / \gamma_{b}\right) / 2 .
\end{gathered}
$$

In practice the $\mathrm{rf}$ term, which is a function only of $\alpha$, is nearly constant with a slight oscillation around the value 1.06 all over the range $1 / 2<\alpha<3$, as shown in Ref. [7], for the case of $\mu=\eta=1$. In the following we will therefore take $\Delta p^{\mathrm{rf}}=1.06$.

Finally, the beam exit conditions $\sigma_{2}$ and $\sigma_{2}^{\prime}$ are

$$
\begin{gathered}
\sigma_{2}=\sigma_{r}\left(1+\Delta_{\mathrm{SC}}-\Delta_{B}\right), \\
\sigma_{2}^{\prime}=\sigma_{\mathrm{orb}}^{\prime}-\frac{\gamma^{\prime}}{2 \gamma_{2}} \sigma_{2},
\end{gathered}
$$

where $\sigma_{2}^{\prime}$ is the secular envelope divergence, while $\sigma_{\mathrm{orb}}^{\prime}$ is the actual orbit divergence:

$$
\sigma_{\mathrm{orb}}^{\prime}=\frac{\gamma^{\prime}}{\gamma_{2}} \sigma_{r}\left[1.06+\mu_{\mathrm{SC}}-2 \Delta_{B}\left(1+\Delta_{\mathrm{SC}}-\Delta_{B}\right) / \ln \left(\gamma_{2} / \gamma_{b}\right)\right]
$$

The space-charge impulse factor $\mu_{\mathrm{SC}}$ contains a geometric form factor $\xi(A)$ (see Appendix B), which depends on the bunch aspect ratio $A$ approximately as $\xi(A)=1 /(2.45$ $\left.+1.82 A^{5 / 4}-0.55 A^{3 / 2}\right)$.

According to the normalization applied in Sec. IV to transform the transverse beam size $\sigma$ into the dimensionless quantity $\tau$, defined as $\tau=\sigma / \sqrt{S}$ with $S=I / 2 I_{0} \gamma^{\prime 2} \gamma_{2}$, we give here the corresponding quantities $\tau_{2}=\sigma_{2} / \sqrt{S}$ and $\dot{\tau}_{2}$ $=\gamma_{2} \sigma_{2}^{\prime} / \gamma^{\prime} \sqrt{S}$. Using the quantity $\Lambda$ defined in Sec. V, $\Lambda$ $=I / \gamma^{\prime 2} \sigma_{r}^{2}$, the dimensionless beam conditions $\tau_{2}, \dot{\tau}_{2}$, and $\tau_{2}^{\text {orb }}$ become: 


$$
\begin{gathered}
\tau_{2}=\left(\frac{2 I_{0} \gamma_{2}}{\Lambda}\right)^{1 / 2}\left\{1+\left(\mu+\frac{\pi \zeta(A) \Lambda}{4 I_{0}}\right)\left[1-\frac{\ln \left(\gamma_{2}\right)}{\gamma_{2}-1}\right]-b^{2} \ln ^{2}\left(\gamma_{2} / \gamma_{b}\right) / 2\right\} \\
\dot{\tau}_{2}=\left(\frac{2 I_{0} \gamma_{2}}{\Lambda}\right)^{1 / 2}\left\{0.56-\frac{1}{2}\left[1-\frac{\ln \left(\gamma_{2}\right)}{\gamma_{2}-1}\right]+\frac{\pi \zeta(A) \Lambda}{8 I_{0}}\left(1+\frac{\ln \left(\gamma_{2}\right)}{\gamma_{2}-1}\right)+b^{2} \ln ^{2}\left(\frac{\gamma_{2}}{\gamma_{b}}\right) / 4-b^{2} \ln \left(\frac{\gamma_{2}}{\gamma_{b}}\right)\left[1+\left(\mu+\frac{\pi \zeta(A) \Lambda}{4 I_{0}}\right)\right.\right. \\
\left.\left.\times\left(1-\frac{\ln \left(\gamma_{2}\right)}{\gamma_{2}-1}\right)-b^{2} \ln \left(\frac{\gamma_{2}}{\gamma_{b}}\right) / 2\right]\right\}, \\
\dot{\tau}_{2}^{\text {orb }}=\left(\frac{2 I_{0} \gamma_{2}}{\Lambda}\right)^{1 / 2}\left\{1.06+\frac{\pi \zeta(A) \Lambda}{4 I_{0}}-b^{2} \ln \left(\frac{\gamma_{2}}{\gamma_{b}}\right)\left[1+\left(\mu+\frac{\pi \zeta(A) \Lambda}{4 I_{0}}\right)\left(1-\frac{\ln \left(\gamma_{2}\right)}{\gamma_{2}-1}\right)-b^{2} \ln ^{2}\left(\frac{\gamma_{2}}{\gamma_{b}}\right) / 2\right]\right\}
\end{gathered}
$$

which are functions of only four parameters: $\alpha, A, \Lambda$, and $b$ (recalling that $\gamma_{1}, \gamma_{2}$, and $\gamma_{b}$ are functions only of $\alpha$ ).

Let us assume now that the first half cell may be different, in length, from an exact quarter of rf wavelength, so that the first iris is located at $z_{1}=(1+d) \lambda / 4$ and the second one at $z_{2}=z_{1}+\lambda / 2$. Following the calculations by Serafini [26] we can express the beam energies $\gamma_{1}$ and $\gamma_{2}$ in the form

$$
\gamma_{1}=1+\frac{\pi \alpha}{2}\left[1+\frac{5}{4} d-\left(\frac{5}{16}+\frac{\pi^{2}}{24}\right) d^{2}\right], \quad \gamma_{2}=1+\frac{3 \pi \alpha}{2}\left[1+\frac{5}{12} d-\left(\frac{5}{2}+\frac{\pi^{2}}{3}\right) \frac{d^{2}}{24}\right],
$$

while the term $\Delta p^{\mathrm{rf}}$ and $\Delta_{\mathrm{SC}}$ become

$$
\begin{gathered}
\Delta p^{\mathrm{rf}}=\left\{\mu\left[1+\mu-\frac{\mu \ln \left(\gamma_{1}\right)}{\gamma_{1}-1}\right]-\frac{\eta\left(1-(\alpha / 4 !)^{2}\right) \ln \left(\gamma_{2}\right)}{8}\left[1+\mu-\frac{\mu \ln \left(\gamma_{1} \sqrt[3]{\gamma_{2}}\right)}{2\left(\gamma_{1}-1\right)}\right]\right\}\left(1+\frac{2}{3} d-\frac{d^{2}}{2}\right), \\
\Delta_{\mathrm{SC}}=\left[\mu(1+0.2475 d)+\mu_{\mathrm{SC}}\right]\left[1-\frac{\ln \left(\gamma_{2}\right)}{\gamma_{2}-1}\right] .
\end{gathered}
$$

The actual orbit divergence at the second iris, $\sigma_{\mathrm{orb}}^{\prime}$, is found to be

$$
\sigma_{\mathrm{orb}}^{\prime}=\frac{\gamma^{\prime}}{\gamma_{2}} \sigma_{r}\left[1.06\left(1+\frac{2}{3} d-\frac{d^{2}}{2}\right)+\mu_{\mathrm{SC}}-2 \Delta_{B}\left(1+\Delta_{\mathrm{SC}}-\Delta_{B}\right) / \ln \left(\gamma_{2} / \gamma_{b}\right)\right] .
$$

Finally, the dimensionless beam exit conditions $\tau_{2}$ and $\dot{\tau}_{2}^{\text {orb }}$ at the second iris are

$$
\tau_{2}=\left(\frac{2 I_{0} \gamma_{2}}{\Lambda}\right)^{1 / 2}\left\{1+\left(\mu(1+0.2475 d)+\frac{\pi \zeta(A) \Lambda}{4 I_{0}}\right)\left[1-\frac{\ln \left(\gamma_{2}\right)}{\gamma_{2}-1}\right]-b^{2} \ln ^{2}\left(\gamma_{2} / \gamma_{b}\right) / 2\right\},
$$

and

$$
\begin{aligned}
\dot{\tau}_{2}^{\text {orb }}= & \left(\frac{2 I_{0} \gamma_{2}}{\Lambda}\right)^{1 / 2}\left\{1.06\left(1+\frac{2}{3} d-\frac{d^{2}}{2}\right)+\frac{\pi \zeta(A) \Lambda}{4 I_{0}}-b^{2} \ln \left(\frac{\gamma_{2}}{\gamma_{b}}\right)\left[1+\left(\mu(1+0.2475 d)+\frac{\pi \zeta(A) \Lambda}{4 I_{0}}\right)\left(1-\frac{\ln \left(\gamma_{2}\right)}{\gamma_{2}-1}\right)\right.\right. \\
& \left.\left.-b^{2} \ln ^{2}\left(\frac{\gamma_{2}}{\gamma_{b}}\right) / 2\right]\right\} .
\end{aligned}
$$

\section{APPENDIX B}

The transverse rms kick due to the space-charge field is represented by the factor $\mu_{\mathrm{SC}}$ in Eq. (A2) for a Gaussian distribution of transverse size $\sigma_{r}$ and longitudinal $\sigma_{z}$, with total charge $Q_{b}$. As extensively reported elsewhere [7], $\mu_{\mathrm{SC}}$ is calculated by averaging (in the rms sense) the transverse electric field component of the bunch $E_{r}^{\mathrm{SC}}$ (at rest in the laboratory frame),

$$
E_{r}^{\mathrm{sc}}(r, \zeta)=\frac{Q}{2 \epsilon_{0}(2 \pi)^{3 / 2} \sigma_{r}^{2} \sigma_{z}} r \int_{0}^{\infty} d x \frac{\exp \left\{-(1 / 2)\left[r^{2} / \sigma_{r}^{2}(1+x)+\zeta^{2} / \sigma_{z}^{2}\left(1+A^{2} x\right)\right]\right\}}{(1+x)^{2} \sqrt{\left(1+A^{2} x\right)}}
$$

over the charge density distribution

$$
\rho(r, \zeta)=\frac{Q_{b}}{(2 \pi)^{3 / 2} \sigma_{r}^{2} \sigma_{z}} \exp \left[-\frac{r^{2}}{2 \sigma_{r}^{2}}-\frac{\zeta^{2}}{2 \sigma_{z}^{2}}\right]
$$


to get

$$
\mu_{\mathrm{SC}}=\frac{\pi \gamma_{2}}{2 E_{0} \gamma^{\prime} \sigma_{r}}\left[\frac{1}{2 Q} \iint_{V_{\infty}} \int \rho(r, \zeta) E_{r}^{2}(r, \zeta) r d r d \phi d \zeta\right]^{1 / 2},
$$

which can be cast in the form $\mu_{\mathrm{SC}}=\pi I \xi(A) / 4 I_{0} \gamma^{\prime 2} \sigma_{r}^{2}$, with

$$
\xi(A)=\left\{\int_{0}^{\infty} d x_{1} \int_{0}^{\infty} d x_{2} \frac{\left[\left(1+A^{2} x_{1}\right)\left(1+A^{2} x_{2}\right)+2+A^{2}\left(x_{1}+x_{2}\right)\right]^{-1 / 2}}{\left[\left(1+x_{1}\right)\left(1+x_{2}\right)+2+x_{1}+x_{2}\right]^{2}}\right\}^{1 / 2} .
$$

$\xi(A)$ can be represented within $1 \%$ error in the range $0 \leqslant A \leqslant 6$ by the function

$$
\xi(A)=\frac{1}{2.45+1.82 A^{5 / 4}-0.55 A^{3 / 2}} .
$$

Since $\mu_{\mathrm{SC}}$ is actually the global rms space-charge kick on the bunch, we are interested in evaluating the kick $\mu_{\mathrm{SC}}^{+}$applied on the central bunch slice, located at $\zeta=0$, and the one $\mu_{\mathrm{SC}}^{-}$applied to the slice located at $\zeta=\sigma_{z}$. These are given by

$$
\mu_{\mathrm{SC}}^{+}=\frac{\pi \gamma_{2}}{2 E_{0} \gamma^{\prime} \sigma_{r}}\left[\sqrt{\frac{\pi}{2}} \frac{\sigma_{z}}{Q} \int_{0}^{2 \pi} d \varphi \int_{0}^{\infty} \rho(r, 0) E_{r}^{2}(r, 0) r d r\right]^{1 / 2}
$$

and

$$
\mu_{\mathrm{SC}}^{-}=\frac{\pi \gamma_{2}}{2 E_{0} \gamma^{\prime} \sigma_{r}}\left[\left(\frac{\pi}{2}\right)^{1 / 2} \frac{\sigma_{z}}{Q} \int_{0}^{2 \pi} d \varphi \int_{0}^{\infty} \rho\left(r, \sigma_{z}\right) E_{r}^{2}\left(r, \sigma_{z}\right) r d r\right]^{1 / 2}
$$

which can be cast in the form $\mu_{\mathrm{SC}}^{+}=\pi I \xi^{+}(A) / 4 I_{0} \gamma^{\prime 2} \sigma_{r}^{2}$ and $\mu_{\mathrm{SC}}^{-}=\pi I \xi^{-}(A) / 4 I_{0} \gamma^{\prime 2} \sigma_{r}^{2}$, with

$$
\xi^{+}(A)=\left\{\int_{0}^{\infty} d x_{1} \int_{0}^{\infty} d x_{2} \frac{\left[\left(1+A^{2} x_{1}\right)\left(1+A^{2} x_{2}\right)\right]^{-1 / 2}}{\left[\left(1+x_{1}\right)\left(1+x_{2}\right)+2+x_{1}+x_{2}\right]^{2}}\right\}^{1 / 2}
$$

and

$$
\xi^{-}(A)=\left\{\int_{0}^{\infty} d x_{1} \int_{0}^{\infty} d x_{2} \frac{\exp \left\{-\left[-1-1 /\left(1+A^{2} x_{1}\right)-1 /\left(1+A^{2} x_{2}\right)\right] / 2\right\}}{\left[\left(1+x_{1}\right)\left(1+x_{2}\right)+2+x_{1}+x_{2}\right]^{2}\left[\left(1+A^{2} x_{1}\right)\left(1+A^{2} x_{2}\right)\right]^{1 / 2}}\right\}^{1 / 2} .
$$

It is convenient to redefine the kicks $\mu_{\mathrm{SC}}^{+}$and $\mu_{\mathrm{SC}}^{-}$in terms of rescaled currents $I^{+}$and $I^{-}: \mu_{\mathrm{SC}}^{+}$ $=\pi I^{+}(A) \xi(A) / 4 I_{0} \gamma^{\prime 2} \sigma_{r}^{2}$ and $\mu_{\mathrm{SC}}^{-}=\pi I^{-}(A) \xi(A) / 4 I_{0} \gamma^{\prime 2} \sigma_{r}^{2}$, where

$$
I^{+}(A)=I \frac{2.45+1.82 A^{5 / 4}-0.55 A^{3 / 2}}{1.84+1.95 A^{5 / 4}-0.65 A^{3 / 2}}
$$

and

$$
I^{-}(A)=I \frac{2.45+1.82 A^{5 / 4}-0.55 A^{3 / 2}}{3.84+1.74 A^{5 / 4}-0.34 A^{3 / 2}}
$$

are valid approximations for $I^{+}$and $I^{-}$in the range $0 \leqslant A \leqslant 6$. At $A=1$ we have $I^{+}=1.19 I$ and $I^{-}=0.71 I$.

In order to calculate the geometrical form factor $g(\zeta)$ we consider here only the linear component $E_{\operatorname{lin}}(\zeta)$ of the space charge field in Eq. (B1), i.e.,

$$
g(\zeta)=E_{\operatorname{lin}}(\zeta)=\int_{0}^{\infty} d x \frac{e^{-(1 / 2) \zeta^{2} / \sigma_{z}^{2}\left(1+\bar{A}^{2} x\right)}}{(1+x)^{2} \sqrt{\left(1+\bar{A}^{2} x\right)}}
$$

which is the source of the perveance term $\kappa_{s}(\zeta)$ in the envelope equation; we want to study its dependence on the slice position $\zeta$ for low aspect ratios $\bar{A}=A / \gamma$ in the rest reference frame. For highly relativistic beams, i.e., $\gamma \gg 1$ the transverse space-charge-field dependence versus the longitudinal position $\zeta$ resembles the behavior of the charge density distribution: in fact, we have

$$
g(\zeta) \underset{\bar{A} \rightarrow 0}{\longrightarrow} \exp \left[-\zeta^{2} / 2 \sigma_{z}^{2}\right],
$$


as expected. For small $\bar{A}$ we approximate $g(\zeta)$ with its Taylor expansion up to second order in $\bar{A}$ around $\bar{A}=0$, to obtain

$$
g(\zeta)=e^{-\zeta^{2} / 2 \sigma_{z}^{2}}\left\{1+\bar{A}^{2}\left[\left(1-\frac{\zeta^{2}}{\sigma_{z}^{2}}\right)\left(\frac{1}{2}+\ln \bar{A}\right)-1\right]\right\}+O\left(\bar{A}^{4}\right)
$$

[1] J. S. Fraser et al., IEEE Trans. Nucl. Sci. 32, 1791 (1985).

[2] C. Travier, Part. Accel. 36, 33 (1991).

[3] X. Qiu et al., Phys. Rev. Lett. 76, 3723 (1996).

[4] B. E. Carlsten, Nucl. Instrum. Methods A 285, 313 (1989).

[5] J. D. Lawson, The Physics of Charged Particle Beams, 2nd ed. (Oxford University Press, New York, 1988).

[6] J. B. Rosenzweig and L. Serafini, Phys. Rev. E 49, 1599 (1994); S. C. Hartman and J. B. Rosenzweig, ibid. 47, 2031 (1993).

[7] L. Serafini, Part. Accel. 49, 253 (1995).

[8] P. Lapostolle, IEEE Trans. Nucl. Sci. NS-18, 1101 (1971).

[9] K. J. Kim, Nucl. Instrum. Methods A 275, 201 (1989).

[10] J. Coacolo (private communication).

[11] L. Serafini, R. Zhang, and C. Pellegrini, Nucl. Instrum. Methods A 387, 305 (1997).

[12] J. M. Dolique and J. C. Coacolo, Nucl. Instrum. Methods A 340, 231 (1994).

[13] L. Serafini and C. Pagani, Proceedings of the European Particle Accelerator Conference (World Scientific, Singapore, 1988), p. 866.

[14] The current density $J$ is not the real beam current density along acceleration: it is just a reference value calculated with the beam spot size at the cathode $\sigma_{r}$.

[15] C. Travier, Ph.D. thesis, Université De Paris Sud, Orsay-LAL, 1995 (unpublished).
[16] J. B. Rosenzweig et al., Nucl. Instrum. Methods A 341, 379 (1994).

[17] J. B. Rosenzweig and E. Colby, in Advanced Accelerator Concepts, edited by P. Schoessow, AIP Conf. Proc. No. 335 (AIP, New York, 1995), p. 724.

[18] M. Reiser, Theory and Design of Charged Particle Beams (John Wiley \& Sons, New York, 1994), p. 201.

[19] O. A. Anderson, Part. Accel. 21, 197 (1987).

[20] E. Colby, J.-F. Ostiguy, and J. B. Rosenzweig, in Advanced Accelerator Concepts (Ref. [17]), p. 708.

[21] D. T. Palmer et al., in Proceedings of the 1995 IEEE Particle Accelerator Conference, IEEE Cat. No. 95CH35843, 1996, p. 2432.

[22] D. C. Nguyen et al., Nucl. Instrum. Methods A 341, 29 (1994).

[23] Taking indeed a $100 \mathrm{~A}$ beam at $19 \mathrm{MeV}$, the beam spot at the photoinjector exit, as predicted by the invariant envelope mode, is $\sigma=0.9 \mathrm{~mm}$, so that Eq. (6.1) predicts a spot size increase of less than $125 \mu \mathrm{m}$ over $1-\mathrm{m}$ drift.

[24] R. Sheffield (private communication).

[25] J. B. Rosenzweig et al., in Advanced Accelerator Concepts (Ref. [17]), p. 684.

[26] L. Serafini, in Advanced Accelerator Concepts, edited by J. S. Wurtele, AIP Conf. Proc. No. 279 (AIP, New York, 1993), p. 645 . 